This item was submitted to Loughborough's Research Repository by the author.

Items in Figshare are protected by copyright, with all rights reserved, unless otherwise indicated.

\title{
Repair: comparing Facebook 'chat' with spoken interaction
}

PLEASE CITE THE PUBLISHED VERSION

http://dx.doi.org/10.1177/1750481313510815

PUBLISHER

Sage Publications / (c) The Author(s)

VERSION

AM (Accepted Manuscript)

PUBLISHER STATEMENT

This work is made available according to the conditions of the Creative Commons Attribution-NonCommercialNoDerivatives 4.0 International (CC BY-NC-ND 4.0) licence. Full details of this licence are available at: https://creativecommons.org/licenses/by-nc-nd/4.0/

\section{LICENCE}

CC BY-NC-ND 4.0

\section{REPOSITORY RECORD}

Meredith, Joanne, and Elizabeth Stokoe. 2013. "Repair: Comparing Facebook 'chat' with Spoken Interaction". Loughborough University. https://hdl.handle.net/2134/18111. 


\title{
Paper for DISCOURSE \& COMMUNICATION
}

\author{
Repair: Comparing Facebook 'chat' with spoken \\ interaction
}

Joanne Meredith and Elizabeth Stokoe

Department of Social Sciences

Loughborough University

Loughborough

LE11 3TU

UK

Published in Discourse \& Communication

2014, Vol. 8(2) 181-207

DOI: $10.1177 / 1750481313510815$ 


\section{Biographical Information}

Jo Meredith is a postgraduate research student in the Department of Social Sciences at Loughborough University, UK. Her current research uses conversation analysis to explore whether online written interactions are empirically different from spoken interactions.

Elizabeth Stokoe is Professor of Social Interaction in the Department of Social Sciences at Loughborough University, UK. Her current research interests are in conversation analysis, membership categorization, and social interaction in various ordinary and institutional settings, including neighbour mediation, police interrogation, university student interaction, role-play and simulated interaction. She is the author of Discourse and Identity (with Bethan Benwell, Edinburgh University Press, 2006) and co-editor of Conversation and Gender (with Susan Speer, Cambridge University Press, 2011). 


\title{
Repair: Comparing Facebook 'chat' with spoken
}

\section{interaction}

\author{
Abstract \\ Previous research on the conversation analytic phenomenon of 'repair' has focused on \\ its design and function in spoken interaction. Conversely, research on written text or \\ writing rarely focuses on interaction. In this paper, we examine repair in written \\ discourse; specifically in online settings. The data corpus comprises one-to-one quasi- \\ synchronous Facebook 'chat'. First, we show that, as in spoken interaction, repair \\ happens. This basic observation supports conversation analytic arguments that features \\ of talk, like repair and laughter, do not 'leak randomly' into interaction but are \\ precision-timed and designed to accomplish action. Second, we report on two types of \\ repair: visible repair which can be seen and oriented to by both participants in the \\ interaction, and message construction repair, which is available only to the message's \\ writer. While the practice of message construction repair is made possible through the \\ affordances of the online medium, it nevertheless shows how participants in written \\ interaction are oriented to the same basic contingencies as they are in spoken talk: \\ building sequentially organized courses of action and maintaining intersubjectivity. We \\ suggest that assumptions about differences between spoken and online interaction are
}


premature. Rather, we argue that online interaction should be treated as an adaptation of an oral speech-exchange system.

\section{Keywords}

Facebook, repair, online, conversation analysis; screen-capture methods 


\section{Introduction}

This paper examines the conversation analytic phenomena of 'repair' and 'correction' in online interaction. The focus is specifically on self-initiated self-repair and selfcorrection, in which writers both initiate and complete the repair themselves. Most previous research on repair has focused on spoken, rather than written, interaction. The current paper analyses written interaction from Facebook 'chat' between friends and intimates. First, we present a brief summary of research on language and the internet, before giving an overview of existing work on repair and correction. In the analysis that follows, we make a distinction between visible and message construction repairs, and how the affordances of the internet make such a distinction possible. By visible repairs, we mean repairs which are 'seeable' by all parties to the interaction. By message construction repair, we mean repairs that occur in turn construction, which are unavailable to the recipient. Message construction repair allows us to examine the way participants construct and modify the actions their turns are designed to accomplish.

A key aim of the paper is to consider the similarities and differences between the forms and types of repair found in spoken and online conversation. In so doing, we identify some specific affordances of online interaction, which are often discussed in the literature on online discourse but rarely based in empirical work. We propose that online interaction is as a particular speech-exchange system, where 'interaction differs from 
ordinary conversation in systematic ways' (Hester and Francis, 2000: 392, emphasis in original). Through the investigation of repair, we will discuss whether online interaction differs in systematic ways from spoken interaction.

\section{Language and the internet}

The language of the internet is an ever-growing topic for research, including the discourse of internet forums (Brown, 2009; Holtz and Wagner, 2009); the interrogation of a distinct internet language (Cherny, 1999; Crystal, 2001), and the practices of online interaction (e.g. Benwell and Stokoe, 2006; Greenfield and Subrahmanyam, 2003; Herring, 1999; Werry, 1996). There are three broad trajectories of work on 'asynchronous' and 'quasi-synchronous' interaction. In asynchronous interaction, participants are not necessarily online at the same time: one party posts a message and another reads and responds to it whenever they want to. Responses can, therefore, be delayed for hours or days; in fact, indefinitely. A key feature of this online medium is that participants often use online pseudonyms rather than their real (offline) names, although they sometimes appear to know each other in the offline world. Studies of asynchronous interaction include the analysis of online forums, focused mainly on the topical content and narrative accounts of forum posts (e.g. Horne and Wiggins, 2009; Lamerichs and te Molder, 2003; Sneijder and te Molder, 2005). Some authors have 
examined this kind of data from an interactional perspective, analyzing the construction of opening posts (Antaki et al., 2005); how opening posts are responded to (Vayreda and Antaki, 2009); turn-taking practices (Gibson, 2009), and how forum rules are oriented to by posters (Stommel, 2008; Stommel and Koole, 2010). Much of this research treats online interactions as if they were merely 'everyday interaction' (Flinkfeldt, 2011) and analyze them as such.

A second focus has been on how people interact in chatrooms. Both chatrooms and forums are multi-party interactions between participants who do not know each other in the offline world prior to joining the chatroom, although it is possible that they may meet up as a result of their online interactions (Hutchby, 2001). However, chatroom interaction is quasi-synchronous and, as such, is more similar to spoken conversation because turns are taken more immediately and frequently. However, it is not 'synchronous' because the message production and transmission are separate (Garcia and Jacobs, 1999). Interaction in chatrooms tends to involve multiple (maybe thousands of) participants who are online at the same time. So, researchers have examined how speech-exchange systems in such interactions differ from spoken conversation (Garcia and Jacobs, 1999); how participants make their interactions as 'speech-like' as possible (Werry, 1996); how people have adapted face-to-face conversational practices to interact effectively online (Greenfield and Subrahmanyam, 2003; Rintel et al., 2001), and how sequential and interactional coherence is maintained 
across multiparty interactions (Greenfield and Subrahmanyam, 2003; Herring, 1999). Taken together, this work suggests that, while the interactions are written, they are, nevertheless, sequentially organized in some ways similar to spoken conversation.

The third trajectory of online interaction research is much smaller. Despite its popularity as a method of communication, there are few studies that examine one-to-one quasi-synchronous interaction via instant messaging (e.g., MSN, Skype, or Facebook) between friends, partners, or, more generally, people who already know each other in their offline lives (although the nature of the relationship between participants is, of course, an empirical question). The research that has been done has focused on taskoriented interaction between colleagues, such as meetings (Markman, 2009) or information-seeking chats (Epperson and Zemel, 2008). However, there is little work on chat between intimates.

Exceptions to this include Rawclaw (2008) who examined closings in instant messaging chats between friends. He noted that the closing sequences often closely mirrored those in spoken interaction. However, users occasionally made use of the affordances of the medium - in this case, the ability to trigger an automatic ‘unavailability' message - to curtail the closing sequence. Berglund (2009) also examined chats on a variety of different instant messaging services. As with the research on multi-party interactions, she focused on coherence and turn-adjacency. However, in both these studies, the chat 'transcripts' contained no information about 
turn production, so there was no 'information about when the other person starts typing' (Berglund, 2009: 10). Furthermore, there was 'no way of knowing whether participants do manage to pay attention to information appearing on screen while preparing their own messages, other than by investigating their contributions to the subsequent interaction' (Berglund, 2009: 10). The current study, in contrast, addresses such limitations by basing its analysis on screen capture videos of both instant-message construction and sending, as well as a timed transcript of the entire interaction between parties.

Repair and correction

The focus of this paper is on a much-studied feature of spoken conversation: 'repair'. This refers to the processes by which speakers deal with troubles which arise in speaking, hearing or understanding talk (Schegloff, 2007). Repair operations reveal how interacting parties construct and maintain intersubjectivity (Schegloff, 2006). A key finding about repair is that it can occur when there is no hearable error, mistake or fault and, equally, 'hearable error does not necessarily yield the occurrence of repair/correction' (Schegloff et al., 1977: 363). Any aspect of talk can become the target of repair, from word selection to prosody, and it is, therefore, an omnirelevant feature of interaction. Consider the following example. 
(1) From Sidnell (2010: 111)

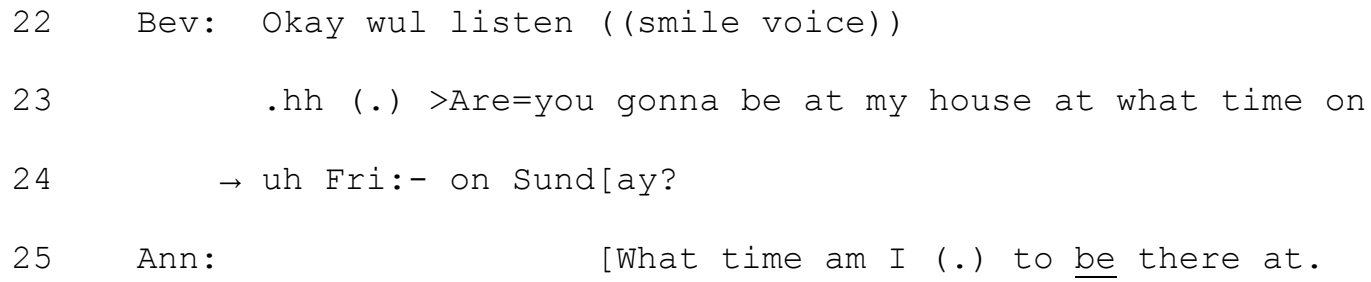

In this example, the trouble-source or repairable item is 'Fri:-' (line 24), which is lengthened and then cut off. Before the trouble-source, the 'uh' indicates that there may be some disjunction with prior talk. Such 'non-lexical speech perturbations' (Schegloff et al., 1977: 367) are common in self-initiated repairs; that is, when the repair is initiated by the current speaker. Extract 1 is an example of self-repair, because the repair is initiated and completed by the same speaker. In producing the repair, Bev repeats the word 'on' which previously appeared at line 23 . This comprises the 'framing' of a repair, when some part of the prior talk is repeated, locating the repairable item (Sidnell, 2010). Repairs are either 'pre-framed', where the talk prior to the trouble-source is repeated, or 'post-framed', where the talk following the trouble-source is repeated. Schegloff et al (1977) found that, empirically, there is a strong preference for selfrepair, mostly because opportunities for self-initiation come before opportunities for other-initiation. Another observation about the preference for self-repair is that others 
typically only initiate repair, and leave it to the speaker of the trouble-source to do the actual repair (Schegloff et al., 1977).

Previous research on self-repair has investigated talk and embodied conduct in face-to-face or telephone conversation (e.g. Hepburn et al., 2012; Lerner and Kitzinger, 2007; Martin and Sahlström, 2010; Wilkinson and Weatherall, 2011). There is little research on repair in online interaction, but what has been done suggests that 'interlocutors adapt the basic repair mechanisms which are available in ordinary conversation to the technical specificities of chat communication' (Schönfeldt and Golato, 2003: 272). Schönfeldt and Golato's (2003) work examined repair in multiparty quasi-synchronous web chats and showed that participants often adjust practices to suit the particular conditions which arise from that web chat. They argue that due to the turn-taking organisation of chats, there are different positions from which repair can be initiated. For example, they describe 'next opportunity space repair' thus:

[I]f I have sent off my turn (i.e. message) to the server, and I realize I would like to change something in it, and I start typing this rephrasing immediately, and I send it off as quickly as possible, there is still no guarantee that my message will be posted before the response of my interlocutor. Thus, the next available opportunity after the same turn (message) repair is the next turn (message) (Schönfeldt and Golato, 2003: 257). 
Equally, they argue that there are trouble-sources which are unique to 'chat' interaction. For example, because there are usually many conversations running concurrently in any one chatroom, participants must ensure that turns designed for particular recipients reach that recipient. If this does not happen then it 'can be targeted with repair or can even be reprimanded' (Schönfeldt and Golato, 2003: 265), as in the following example:

(2) From Schönfeldt and Golato (2003: 266)

$\begin{array}{lll}1 & \text { (195) Nikkee: } & \text { calvi: probably but first I am going to } \\ 2 & \text { move and I am happy about my apartment } \\ 3 & \text { (289) Nikkee: } & \text { calvi: what's going on? } \\ 4 & \text { (317) Calvino: } & \text { nikee: sorry, you are moving? into your } \\ 5 & & \text { own apartment? really? }\end{array}$

As Schönfeldt and Golato (2003: 266) explain:

In line 1, Nikkee announces that she is going to move into her own apartment. When this news announcement does not receive an uptake from the intended addressee after almost 100 other lines of talk have appeared on the computer 
screen, Nikkee performs a sequential repair. In other words, she is pursuing a response from Calvino.

Whether or not line 3 actually constitutes a repair initiator is moot. However, Schönfeldt and Golato claim that such a repair is specific to 'chat' interaction. However, analysis of spoken conversation has also found that 'when, following a sequence-initiating action, a response is missing (or delayed, or inadequate), speakers may use a range of practices for pursuing a response' (Bolden et al., 2012: 138). While self-initiated repairs can be used to pursue a response, the use of sequential-organizational resources to pursue a response is not the same thing as repair.

More recently, Collister (2011) studied chat logs from an online gaming environment, the 'Massively Multiplayer Online Roleplaying Game' called World of Warcraft. Collister suggested that participants used what she calls a '*-repair' in order to 'correct' misspellings. This involves using an asterisk to mark the corrected version of a previously incorrect spelling, as in the following example:

(3) From Collister (2011: 919)

1 Aniko: when I run ot

2 Aniko: out* 
As Collister explains, 'in line 1, the player Aniko mistypes 'ot' instead of 'out'. Immediately following this, he types 'out', with the * indicating that this is a repair' (p. 919). This 'distinct repair morpheme' was used for both self-repair and other-repair.

It is worth noting, however, that this repair-morpheme applies to corrections, rather than repair. In CA, a distinction is drawn between repair and correction, as repair is 'neither contingent upon error, nor limited to replacement' (Schegloff et al., 1977: 363). As Macbeth (2004: 707) explains, 'though repair can entail correction, correction is a lesser domain both conceptually and empirically. Correction premises 'error', yet studies of repair routinely find repairs where no accountable 'error' can be heard'. In other words, correction is a class of repair which occurs when there has been an 'actual' error. There are two domains of error: 'production errors'; that is, errors in the production of a coherent utterance, or 'interactional errors'; that is, errors in speaking 'appropriately' for the recipients of the talk and for the occasion of the exchange (Jefferson, 1974).

This paper develops the small body of previous work on repair and correction in online interactions. First, we focus on visible repairs, which are produced for, and available to, all parties to the interaction. That is, we examine repair sequences in which, although the 'error' could have been fixed before the text appeared online, nevertheless the repair remains visible. We will show that, in the data corpus, these repair operations are invariably related to production errors. We will discuss the 
positioning of these repairs in comparison to examples from spoken interaction, and the difficulties in comparing the sequential position of such repairs. In the second section, we focus on a form of repair and correction which, although in terms of its production has a corresponding form in spoken conversation, it does not, in terms of recipients, have a spoken equivalent and has not, to the best of our knowledge, been studied before. These are message construction repairs, in that they occur, predominantly, while one party is typing their message. While some previous research has mentioned the selfrepair of messages which respond to other messages that appear during message construction (Garcia and Jacobs, 1999; Markman, 2005), the repairs in this paper are those which are self-initiated self-repairs which occur with no intervention from the other participant. We argue that by analyzing message construction repairs, we can show how, as in spoken interaction, 'it is through self-repair that we see speakers orient to what is the appropriate form to do this action in this sequential place" (Drew et al., 2013: 93, original emphasis), and how, in written discourse participants are equally oriented to matters of sequential organization, action formation, and recipient design. We also show that, despite similarities between written and spoken self-repairs, their initiation and completion during message construction provides for a systematic difference between spoken and online interaction.

\section{Data and Method}


The dataset comprises 75 one-to-one instant messaging conversations using the 'chat' applet on Facebook, the social networking site. As the screenshot in Figure 1 shows, this is an applet which appears at the bottom of a Facebook page, and allows 'friends' to interact with each other in real time.

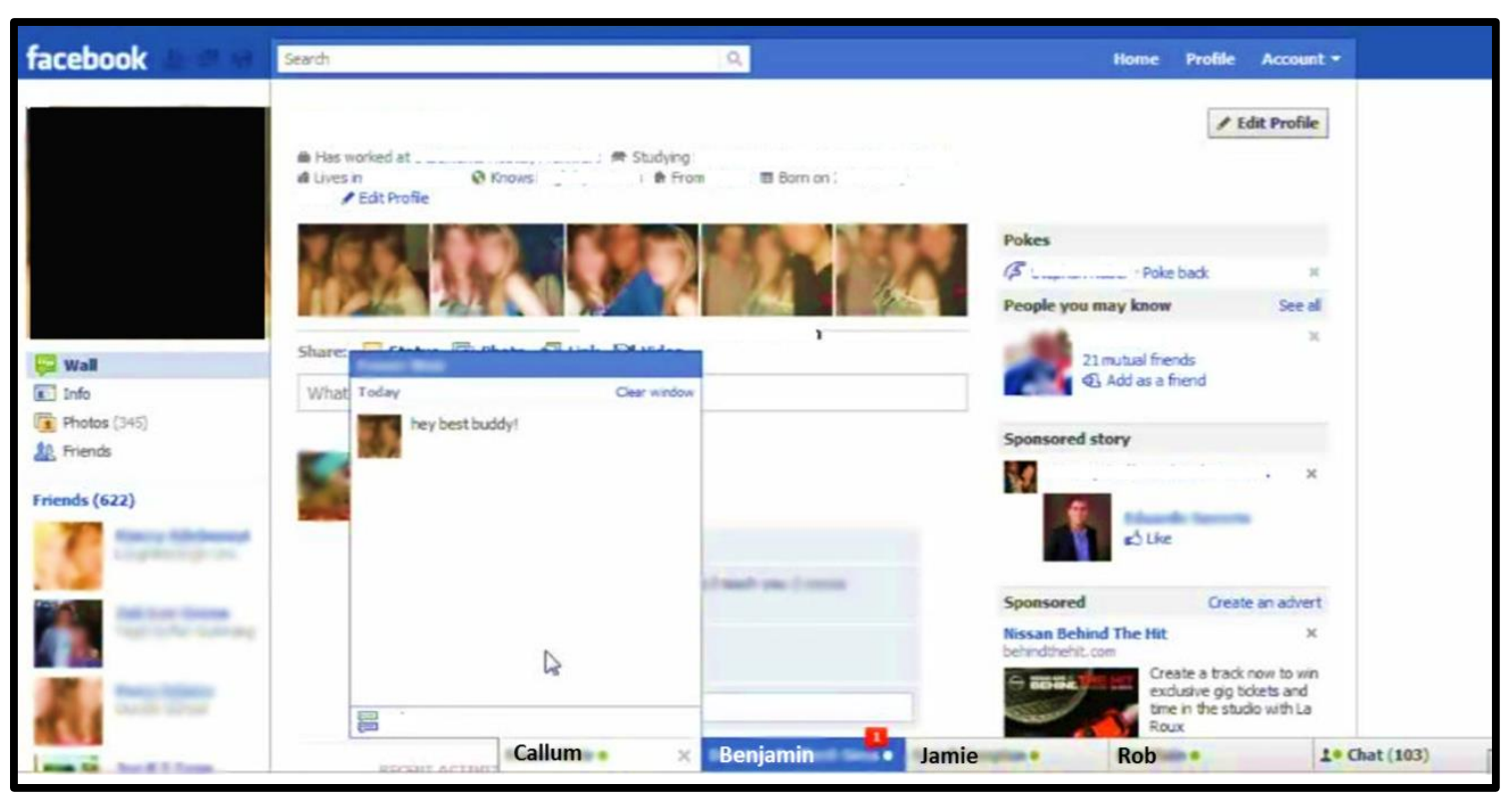

Figure 1. Facebook chat

Here, the participant has a chat window open with Callum, but also has three other ongoing conversations with Benjamin, Jamie and Rob. The participant constructs their message in the box above the other users' name, and once it is sent it appears in the main chat window, and their co-participant can then read it. 
Four participants used software to collect timed transcripts of their Facebook chats and also to record their computer monitor whilst they conducted chats. This provided detailed data about message construction and the management of multiple chats, as well as the other online activities that participants engaged in while chatting. Participants were provided with information on ethical issues such as privacy, anonymity and confidentiality and were able to decide which chats and videos they submitted to the collection. In total the four participants chatted to 33 different people, to whom they provided information about the research and gained their consent. All data, including video data, was anonymized and stored securely.

Once the data was collected, it was transcribed using a system which we adapted from Jefferson's (2004) method for conversation analysis. It includes information to allow readers to understand message construction, gaps between messages, and the management of multiple conversations, as in the following example: ${ }^{1}$

(4) [JM/IS6/F: 42-55]

42

43

44

45
I*: L erm i im mhat was i gonna sya es

0.11 Isla: erm what was i gonna sya

$(2.0)$

I*: she said she ran shit?(.)! :-S es

\footnotetext{
${ }^{1}$ See appendix for full list of transcription symbols
} 


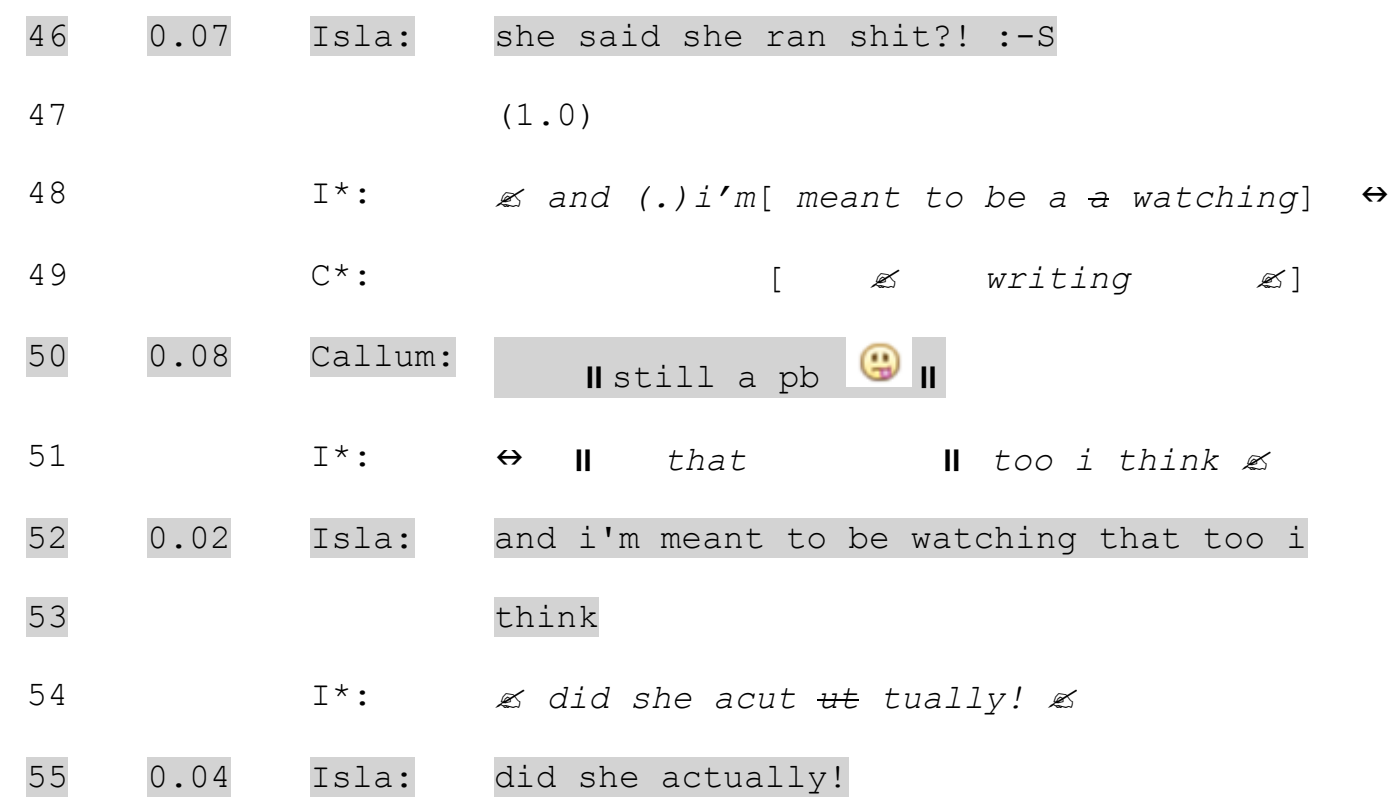

In the above extract, lines $43,46,50,52,53$, and 55, shaded in grey, are the turns which appear in the chat window and thus are visible to recipients. The time given to the left of the participants' name is the time between messages. In line 42 , the $\mathscr{e}$ symbol indicates that this is the construction of the subsequent turn, which is hidden from the recipient. When a participant is constructing their turn they may edit or delete parts of this. Deletions are shown by strikethrough of the letters. We can see this detail in line 42 because Isla is the participant recording her screen. In lines 48-49, an overlap, when both participants are constructing a turn simultaneously, is shown using square brackets. Callum's turn construction is simply marked as 'writing' (line 49) as this is only indicated by a small icon on Isla's screen. We, and Isla, do not have access to what he writes, edits or deletes prior to the message being sent. Once Callum has finished 
writing, he sends the message which appears at line 50. This message appears, in its entirety, at the same time as Isla is writing 'that'. This is denoted by the II II symbols, which indicates that an entire message has appeared at the same time as the other participant is writing a certain word. After Callum posts, Isla continues constructing her

turn at line 51, which is indicated using a double-headed arrow $(\leftrightarrow)$ to show 'latching'. Once transcribed, the data were examined for examples of self-repair in both message construction and message posting.

\section{Analysis}

The analysis is divided into two sections. The first section presents an analysis of visible self-initiated self-repair that all participants to the interaction can see and orient to. We will show that these repairs most often correct a clear error. We will also discuss how such corrections appear sequentially, and how this organization corresponds to spoken interaction. The second section will examine self-initiated self-repair in message construction; that is, repairs which are made as participants construct their messages but are not visible to their recipients. We will observe that and how the online medium provides for such repairs, and also how message construction repairs are performed on both production and interactional errors. We compare these to spoken interaction to show how participants are oriented to the same interactional contingencies in both 
online and spoken interaction. However, in online interaction the repair operation can occur without the recipient having access to it. While we do not attend to otherinitiated repair or other-repair in this paper, such repairs do occur across our materials.

Visible self-initiated self-repair

In the Facebook chat data, repairs occur that are visible to both participants and may be oriented to. Such repairs generally correct an 'error' or 'mistake' and are corrections. These corrections are, most often, both initiated and subsequently repaired by the producer of the trouble-source. In Extract 5, note the visible correction produced by Isla at line 91 .

(5) [JM/IS19/F: 86-91]

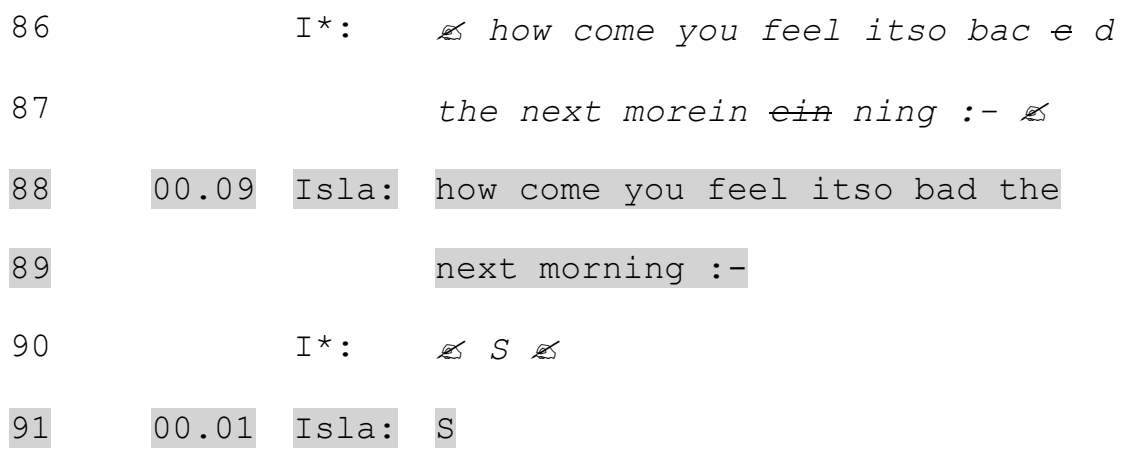


Isla has been discussing Joe's night out the previous night and is asking him why he has such a bad hangover. In line 89 , Isla posts a 'smiley' (':-’). 'Smilies' are used regularly in online and text messaging, built out of standard keyboard punctuation. For example, a colon plus a hyphen plus a bracket constructs a smile face :-). These are used to display the writer's stance towards their turn, sometimes by modifying its action, much like laughter particles and prosodic features do in spoken interaction. In Extract 5, Isla's smiley, once complete, could be seen as indicating confusion about Joe. However, as we can see from line 89 , she has missed off the final letter ' $S$ ' from the smiley, and thus only half of it is posted. It is difficult to know how such a production error was made. It could be that Isla did not notice her error prior to sending, or hit the enter key by mistake. However, even with screen capture data, unless a reason is provided by participants, we cannot say for certain why this error was made. We can, though, see that for the participant this was an error that required fixing. In line 90, Isla types the final letter of the smiley and this subsequently appears in line 91. Thus, Isla corrects her prior turn to avoid ambiguity about her stance, and to enable Joe to respond in a fitted way.

There are similarities between the sequential placement of the repair in Extract 5 and 'transition space repair' in spoken interaction. Turns at talk are constructed from 'turn-constructional-units' (TCUs), and a single turn may comprise any number of TCUs (Sidnell, 2010). At the end of a TCU there is a chance for a transition between 
speakers, the 'transition relevance place' (TRP). Transition space repair occurs when the speaker has potentially completed their TCU but extends their turn to carry out the repair (Liddicoat, 2007), as in the following example:

(6) From Schegloff (1997: 36)

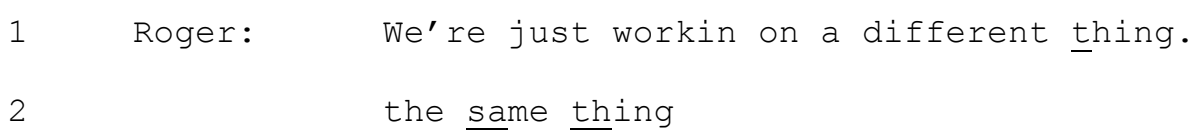

Here, it is the turn-terminal component, 'a different thing', which is to be repaired, and this is done in the transition space; that is 'in the moments just following possible completion of the turn' (Schegloff, 1997: 35). In Extract 5, Isla's repair comes after she has completed a turn and there is potential for her interlocutor to take the next turn. Before he has a chance to do so, though, Isla constructs and posts her repair. Thus, the placement of this correction is similar to a transition space repair, as it is posted in the moments just following the completion of the turn. However, there are problems with describing this as a transition space repair. The notion of a TCU does not fit neatly with online interaction. While TCUs can 'broadly correspond to linguistic categories such as sentences, clauses, single words or phrases' (Hutchby and Wooffitt, 1998: 48), for conversation analysts a TCU is 'essentially anything out of which a legitimate turn has recognizably - for the participants - been built' (Hutchby and Wooffitt, 1998: 48). In 
Extract 5, there is no TRP within the turn; the turn is posted as complete. Isla must therefore take another turn, rather than extending her turn, to do the correction. The repair can more appropriately be termed 'next turn repair'.

In quasi-synchronous chat, the recipient may end up taking a turn before the repair or correction can be formulated. Extract 7 is an example.

(7) [JM/IS6/F: 28-40]

\begin{tabular}{|c|c|c|c|}
\hline 28 & 0.56 & Callum: & (:) Iol im not going to argue with you \\
\hline 29 & & & tonight secret diaries of a call girl \\
\hline 30 & & & coming on lol haha tell her i say \\
\hline 31 & & & hiya and well done \\
\hline 32 & & & for a ew pb $\mathrm{xxx}$ \\
\hline 33 & & $I *:$ & $\leftrightarrow \boldsymbol{a} 3.0$ chatting to $\mathrm{CC} \boldsymbol{\Omega}$ \\
\hline 34 & & & ((Switches to chat with Callum)) \\
\hline 35 & & & $(8.0)$ \\
\hline 36 & & & $\mathscr{L}$ wait wait wait $\mathscr{L s}^{-}$ \\
\hline 37 & 0.15 & Isla: & wait wait wait \\
\hline 38 & & & $(2.0)$ \\
\hline 39 & & $I \star:$ & $\mathscr{L} i \dot{I}\|(2.0)\| \mathbb{L}$ \\
\hline 40 & 0.06 & Callum: & II new II \\
\hline
\end{tabular}


In line 32, Callum has posted 'ew' rather than 'new'. Before his correction is posted (line 40), Isla has already posted a turn at line 37 . This is in contrast to the correction in Extract 5, because there has already been a transition of speakers prior to repair. This is not an example of other-initiated correction, however, because Callum's correction is not responsive to Isla's turn at line 37 . What should, then, have been a next-turn repair, has appeared after the recipient has already posted a response. To some extent this is similar to third turn repair in spoken conversation.

Some participant produces an utterance in a turn which will turn out to be a trouble-source turn...This turn is followed by a contribution from another participant which neither claims nor embodies 'trouble' with what preceded (Schegloff, 1997: 32).

Schegloff notes that, most often, the intervening turn from the other participant is very brief and does not show any trouble in understanding what the speaker meant, as in Extract 8 .

(8) From Schegloff (1997: 32)

1 B: hhh And he's going to make his own paintings,

$2 \mathrm{~A}: \mathrm{Mm}$ hmmm 
Speaker A shows no problem in understanding what B has said, yet B still initiates and repairs their turn at line 3 . The fact that this repair is in the third turn is not by virtue of it being 'relevantly after' (Schegloff, 1997: 34, emphasis in original) the next turn (as would be the case with third position repair) but, rather, the repair is only there incidentally: 'the same repair which would otherwise have been in the transition space now appears in/as third turn' (Schegloff, 1997: 35). So, in Extract 7, Isla's turn at line 37 does not claim nor embody any trouble in understanding Callum's turn at line 28-35. Callum then initiates correction of the trouble-source, but this is not adjacent to the trouble-source turn. Callum's correction may occur after Isla's turn, but it is not relevantly after. It is in third turn only incidentally, because in this type of interaction, Callum does not have access to the construction of Isla's turn.

Thus, self-initiated self-repairs which are visible to both participants in Facebook chat are almost always corrections, but can occur in either next turn or third turn position. This demonstrates that parties are, as with spoken interaction, oriented to the same concerns with maintaining understanding and mutual intelligibility. Comparing repair in spoken and written interaction reveals and specifies further similarities. 
We also found examples of errors, or potential errors, being oriented to by participants but with no subsequent correction. In Extract 9, Katie misspells 'accommodation' at line 3, and while this is oriented to in her next turn, she does not offer a correction.

(9) [JM/KA5/B: 74-76]

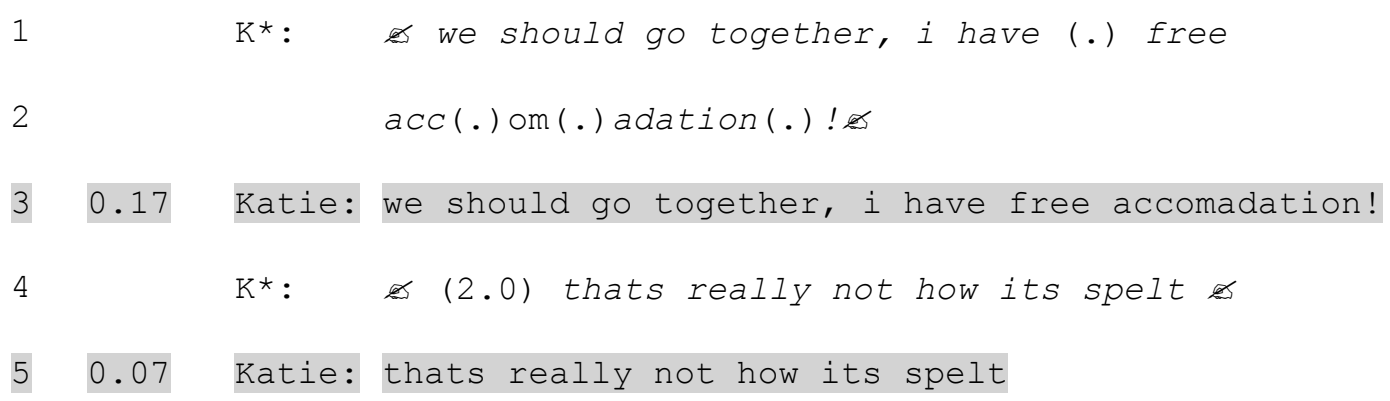

Note the short pauses during the typing of the word 'accomadation', indicating, perhaps as non-lexical speech perturbations do in spoken interaction, that there may be trouble in the formulation of the message. In her next turn Katie orients to this error, but does not correct it. One of the potential affordances of asynchronous online interaction is that users can check their spelling before sending messages. Here, though, is evidence that while users of instant messaging do have time to check spelling, they do not necessarily do so. This suggests that we should not see online affordances as strictly determining the behaviour of users. Rather we should examine how participants actually orient to them or use them (Hutchby, 2003). By orienting to some potential error, Katie is 
attending to what Edwards (2005) refers to as the 'subject-side' or 'speaker-indexical' nature of interaction. That is, when a speaker produces an action, it is 'available for evaluative inferences about the speaker' (Edwards, 2005: 6). Thus, in producing misspellings a negative inference about a participant is made available, and in orienting to the error the participants manage the potential for such inferences.

The analysis presented in section has shown that, and how, the placement of repair in spoken conversation often, but not always, corresponds to those which occur in quasi-synchronous chats. Visible corrections in Facebook chat can appear in next-turn position, but equally can be dislocated from the trouble-source. These are similar to transition space and third turn repairs respectively, but such concepts are difficult to transfer to online interactions due to the differences between TCUs and TRPs in spoken and written interactions. One difference is that the trouble-source is most often one which is specific to written interaction; that is, spelling or typing errors. However, participants do not correct all misspellings, providing evidence that non-standard spelling may be acceptable in online interactions as long as the action is clear. This suggests that participants treat such interaction as informal, despite it being written. These visible corrections of these errors are recipient-designed, dealing with potential misunderstandings and the maintenance of intersubjectivity. For example, in Extract 7, Callum corrects his spelling so that Isla is aware to what he is referring. One clear 
similarity we have found, though, is that the preference for self-correction holds in Facebook chats as much as in spoken conversation.

While we have demonstrated that the sequential position of visible repairs is similar to those in spoken interaction, there is, in contrast, a repair position which is not available in spoken interaction. Turns can be repaired during construction and the recipient is unaware that a repair has occurred. We have termed these repairs message construction repairs, and in the next section we will show how it is interactional errors are most often repaired prior to a message being sent.

Self-initiated self-repair during message construction

In spoken interaction, a variety of trouble-sources can be repaired, including word selection, person reference and action formation (Schegloff et al., 1977; Schegloff, 2013). We found that, in Facebook chat, visible repairs were not performed on these trouble-sources. Instead, they were repaired during message construction, and so not visible to recipients. In the three sections that follow, we examine message construction repairs that attend to issues of person reference, stance, 'pronunciation' and intonation, and more generally, action. As we proceed, we compare these online repairs with examples from spoken interaction. We do this to show how participants in chats are 
oriented to the same interactional contingencies as in spoken interaction, but are able to manage these without the repairs becoming accountable.

\section{(1) Repairs on action formation}

In talk, the projected action of a turn may be repaired as it progresses. In Extract 10, Donny has telephoned Marsha to say that his car has stalled and, as the call progresses, it becomes clear that he is making an implicit request for assistance.

(10) [MDE: Stalled]

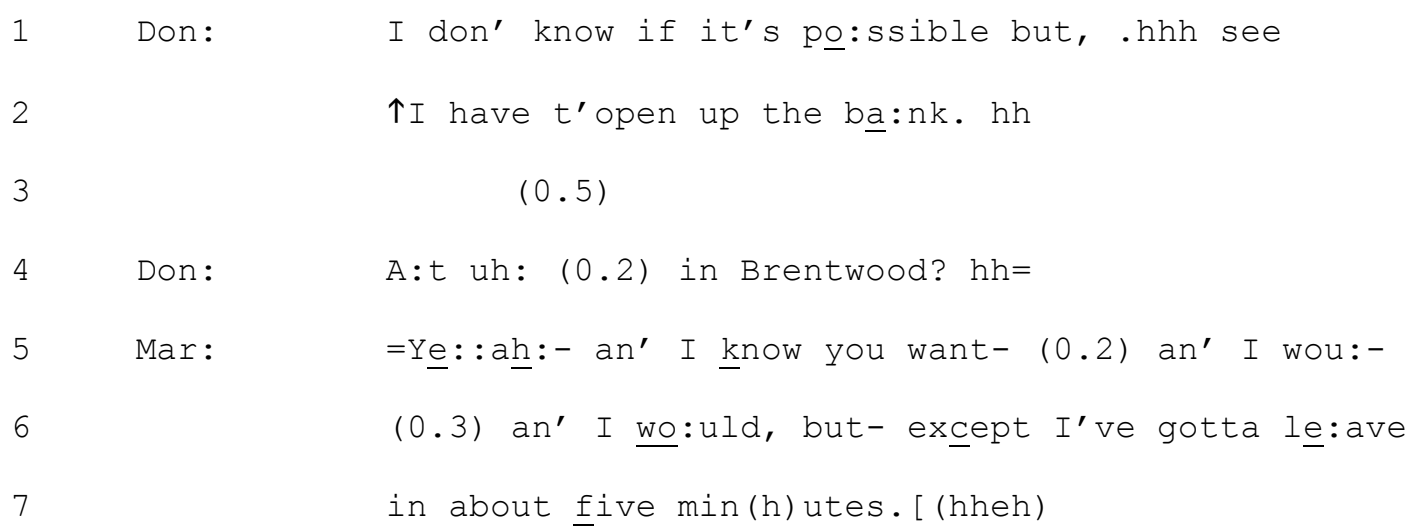

At line 5 Marsha begins a dispreferred response, with an account for - again implicitly - turning down Donny's implicit request. Marsha re-starts her turn three times, but cannot hide these repairs from Donny.

In contrast, consider Extract 11 Isla is moving to close the chat. 
(11) [JM/IS/F: 263-269]

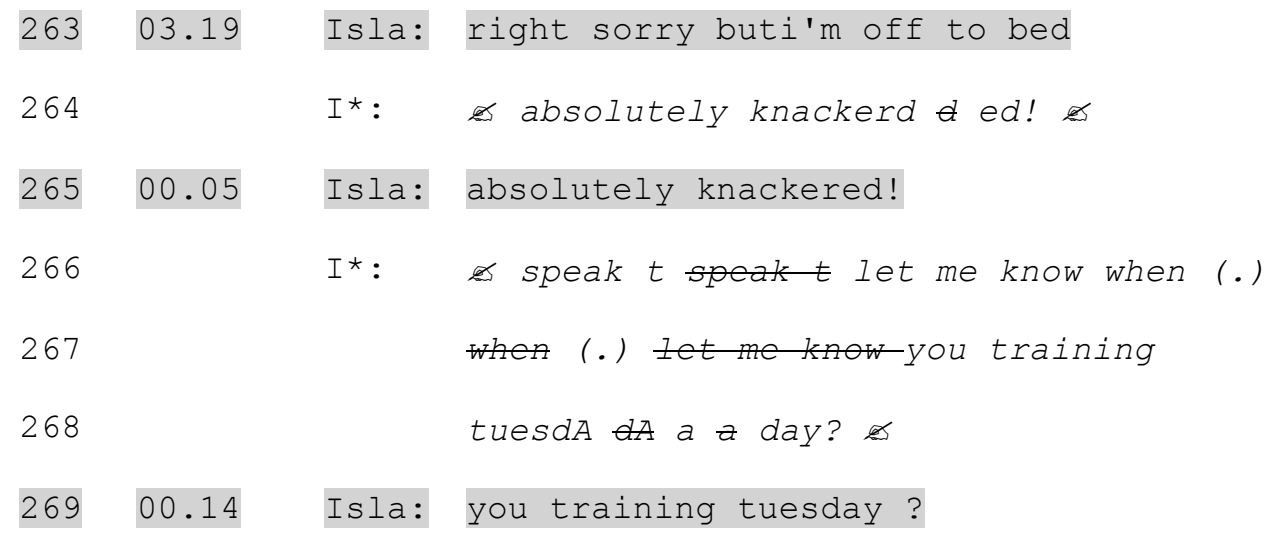

There are a number of corrections in lines 264 and 266-267. These corrections, such as correcting the spelling of 'knackered' in line 264, are also not visible to Isla's recipient. Thus, while visible repairs tend to be corrections, we also find that corrections are made during message construction. We cannot, however, know why a participant corrects some 'typos' in message construction and some after posting. As with spoken interaction, it could just be that self-repairs are initiated and completed in the first opportunity space, where possible, but can also be initiated at later points if necessary. Our main interest is in lines 266-268, which comprise the construction of the message that appears subsequently at line 269. At line 266, Isla deletes the turn beginning 'speak t' and repairs it to 'let me know when'. She then deletes this and 
replaces it with 'you training tuesday?', which is the only part of this message that her recipient sees. By repairing the beginning of her turn, Isla changes its projected action. As turns are constructed they 'project, from their beginnings, aspects of their planned shape and type' (Schegloff, 1987: 71). 'Speak t' may have been headed towards a closing such as 'speak to you soon', which may receive a response which collaboratively moves to close the chat. However, 'let me know when' may project a request for information. The action Isla finally posts is a question which makes an answer relevant, and is not so clearly a pre-closing. Through these concurrent repairs, then, the projected action is repaired from a closing to a request to a question. Like Marsha's repairs, Isla repairs the projected action of her turn. However, Isla's recipient, unlike Donny, remains unaware of these action formation repairs. Isla's repair shows her orientation to how the design of her turn might accomplish a specific action or outcome in projecting a particular response (Hayashi et al., 2013)

Here is another example. Prior to Extract 12, Joe has informed Isla that he has been getting texts from a girl he met the previous night whilst drunk.

(12) [JM/IS19/F: 147-164]

147 00.26 Joe: i didnt do anything i dont think but she 


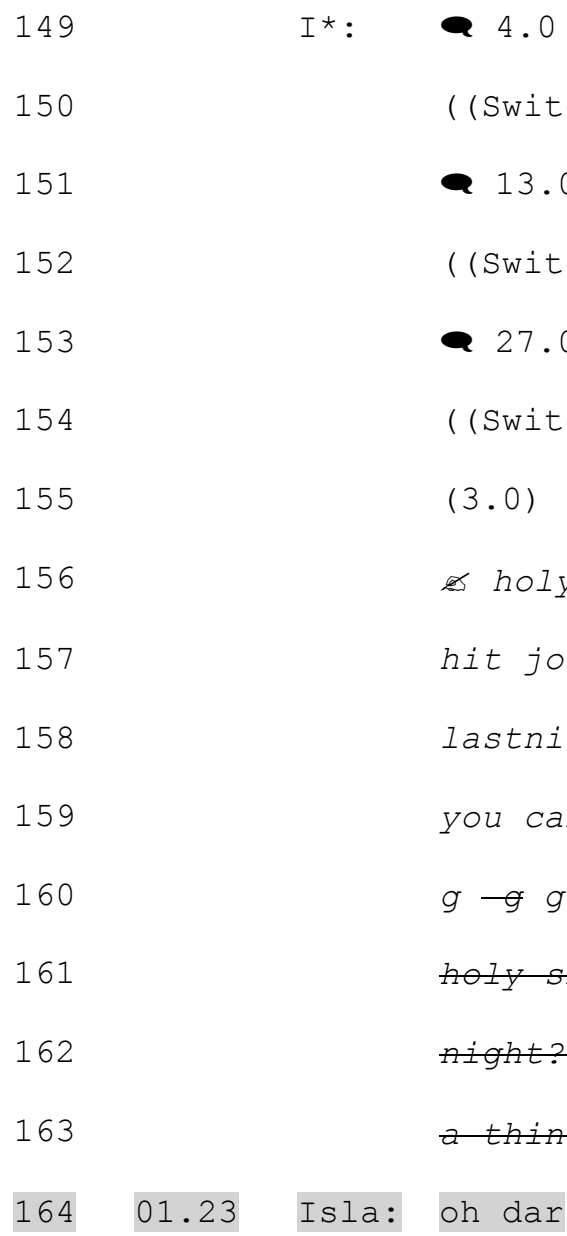

16401.23 Isla: oh dar

Between lines 156-163, Isla is constructing a response to Joe, comprising a question ('where were you last night') and an assertion about his behaviour ('I bet you can't remember...'). However, Isla deletes her original question and assertion, replacing it with an assessment which appears at line 164. Joe is unaware of what was originally written, and only sees what is finally sent at line 164 (which is subsequently corrected to 'oh dear'). 
To understand the interactional significance of this repair we can compare the first, aborted attempt, with what is subsequently sent (Drew et al., 2013). Initially, then, Isla issues an inquiry 'where were you last night?'. The second action is an assertion 'I bet you can't remember a thing lol', which could project a confirmation or denial, or perhaps a humorous account. Either message would have projected a further telling from Joe about his evening. But in her eventual turn Isla does not align herself as an interested recipient (Jefferson, 1978) and does not project further talk on the topic of Joe's night out. While she does not close down the topic explicitly, neither does she invite further talk on it.

Extracts 11 and 12 show that, as with spoken interaction, speakers repair the action-orientation of their talk as it progresses. In Facebook chat participants are able to perform such repairs during message construction, so that the recipient is unaware of it. Here, then, is one interesting observation about the similarities (and differences) between spoken and online interaction: In ordinary spoken interaction, in which turns are formulated instantaneously (unless, perhaps, the speaker is reading a speech or delivering from memory) speakers cannot hide the repairs they make. Repairs may be more or less marked, exposed, and so on (see Stokoe, 2011), but the fact that they are a studyable phenomenon is evidence of their ubiquity in talk. In written interaction such as Facebook chat, participants still formulate and repair turns, but these repairs are completed during message construction prior to the trouble-source occurring in the 
interaction. In contrast to spoken interaction, where repair deals with trouble which is hearable to the recipient, in Facebook chat, message construction repairs attempt to prevent trouble from occurring. However, the placement of these repairs is, to some extent, entirely logical. In spoken interaction, participants make use of the first possible point for initiating repair (Schegloff et al., 1977), and in Facebook chat this is during message construction. It is simply because of the design of Facebook chat that these repairs are not available to the recipient. Our method of capturing live chats has made such an observation available.

\section{(2) Repairs on person reference}

In the previous section, the trouble source or repairable was the actionorientation of the turn. In this section, we discuss repairs on person reference, although these should still be 'understood in terms of the actions they implement' (Lerner and Kitzinger, 2007: 530). 'Person reference' refers to the way speakers refer to themselves and others, and conversation analysts have studied its preference organization, as well as repairs from one type of reference to another (e.g., 'my neighbour', 'the woman down the road', 'Mrs Jones', 'the woman with the long hair', etc.). In the following extract, Scott and Isla are discussing their athletics training.

(13) [JM/IS29/F: 202-205] 


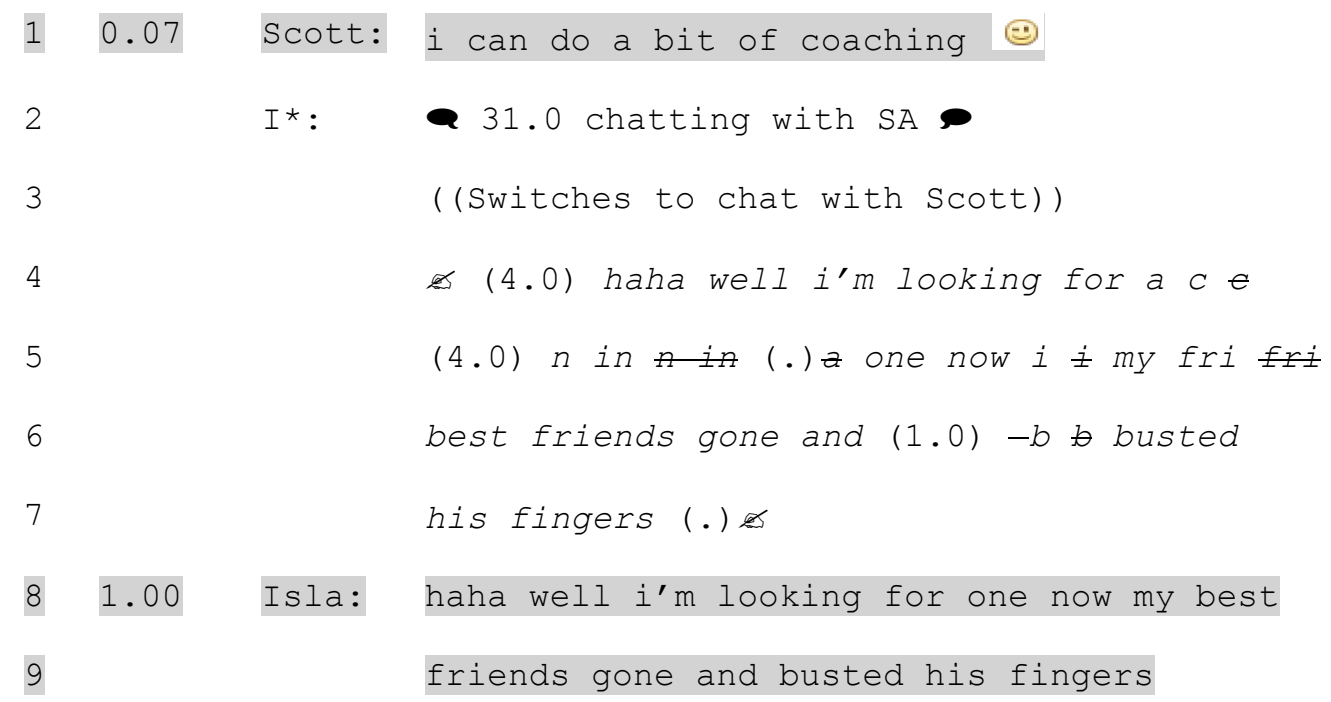

Our target repair occurs between lines 4 and 7, during the construction of the message which appears at lines 8 and 9. Isla begins to type what would presumably be 'a coach' at line 4, but then deletes this and refers it to 'one', which refers indexically to the coach mentioned by Scott in line 1. This demonstrates that, as with spoken interaction, repair can occur when there is no hearable error, mistake or fault (Schegloff et al., 1977). It also shows an orientation to the preference for single, minimal forms of person reference over complex ones (Sacks and Schegloff, 1979). Equally, it orients to the preference for using a locally subsequent reference form (e.g. a pronoun) following an initial full-form reference (Kitzinger et al, 2012). Although in Extract 13 there is no explicit full-form reference to 'a coach', the referent is implicitly available through the verb 'coaching'. 
Isla performs another repair on person reference at line 5, as she repairs 'friend' to 'best friend'. This has a spoken equivalent in 'recalibration repair', where the terms of a formulation are either broadened or narrowed (Lerner et al, 2012). In spoken interaction, when the formulation is narrowed by adding something to the original it can be described as an 'insertion repair' (Wilkinson and Weatherall, 2011), as in the following example.

(14) From Lerner et al., (2012: 196)

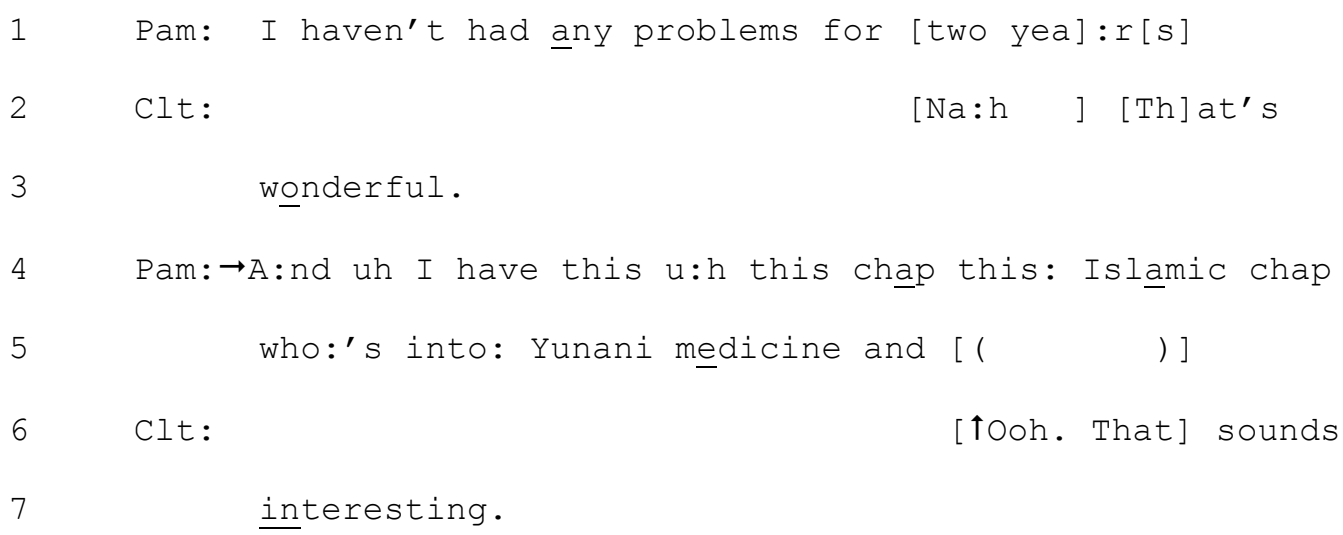

As Lerner et al (2012: 196) note this repair operation 'retains some features of the original formulation but modifies it in a way that adjusts the precision of the reference'. Similarly, in Extract 13 Isla modifies the formulation of 'friend' so that the referent remains the same, it merely becomes a more precise reference of 'best friend'. 
Recalibration repairs are not just repairs; they also reformulate the reference so that it is 'more attuned to the actions, attributes, and setting depicted in talk' (Lerner et al., 2012: 198). When referring to a person, different things can be inferred about the person being referred to, and about the relationship between the speaker and the referent (Lerner and Kitzinger, 2007; Stokoe, 2011). Isla's repair attends to the nature of the relationship between Isla and the referent, in the context of an implicit offer of coaching from Scott (line 1) and implicit acceptance from Isla (lines 8-9). It may also be recipient designed as Isla's 'best friend' may be more recognisable to Scott than the broader referent 'friend'.

The following extract shows an example of the recalibration repair which broadens the referent. Isla and Guy are discussing their living arrangements for the following academic year. There is some difficulty about who will occupy the small room in the shared house they are moving to.

(15) [JM/IS16/B: 233-239]

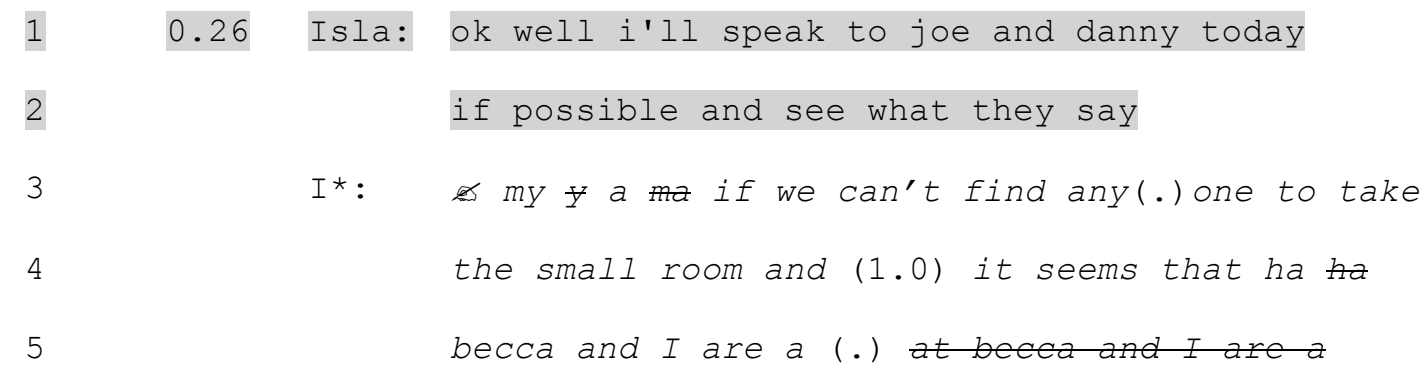


at tow wo of us are going to a a have to

do half a year each tha a en man $n$ ybe (3.0)

joe would reconsider us (2.0) butw we'Il

see 25

if we can't find anyone to take the small room

and it seems that two of us are going to have

to do half a year each then maybe joe

From lines 3-9 Isla is constructing the turn which appears on lines 10-13. At line 5, Isla deletes the phrase 'Becca and I are...' repairing this to 'two of us are...'. The action of this turn remains the same: Isla proposes that two people share the room. However, in the message construction Isla abandons volunteering herself and Becca to take the room, instead suggesting that any two people who will be living in the house might take it. Isla explicitly amends who the referent is, replacing a specific reference with an aggregated one (Lerner et al., 2012). Thus, as with repairs on action, we can see that participants in both spoken and online interactions are concerned with the same interactional preferences for person reference. Participants are, however, able to edit their posts to prevent any trouble for the recipient in locating the referent, rather than managing trouble after it has occurred.

(3) Repairs of 'pronunciation', intonation and stance 
In spoken talk, people use intonation to tell recipients how to hear a word (e.g. in Jefferson's system, 'yes' is different to 'yes::::!'), display their stance towards it, or modify action. Similarly, in online interactions the way a word or phrase is to be read, in terms of its pronunciation, stance, and so on, is indicated through using non-standard spelling (Benwell and Stokoe, 2006).

A speaker's stance can be described as their 'affective treatment of the events he or she is describing' (Stivers, 2008: 37). In other words, a speaker can display their 'attitude' or 'emotion' towards their utterances by, for example, interpolating a word with laughter (e.g. Potter and Hepburn, 2010). Equally, speakers may modify the action of a turn such that recipients understand what sort of response is relevant. In spoken interaction, speakers do this through prosodic features (Local and Walker, 2008), laughter (Jefferson et al., 1987; Potter and Hepburn, 2010) or embodied conduct (Goodwin, 2007). In online interaction, these features are not available; yet the resources of the medium may be used to formulate the same sorts of activities. These stance markers include 'smilies' which, as discussed earlier, are a resource members use to solve the problem of the unavailability of intonation and other embodied conduct.

Consider the following extract from a speed date (see Stokoe, 2010). M has been discussing his job as an actor and the way it helps him 'connect' with people.

(16) [Stokoe: SD-5] 


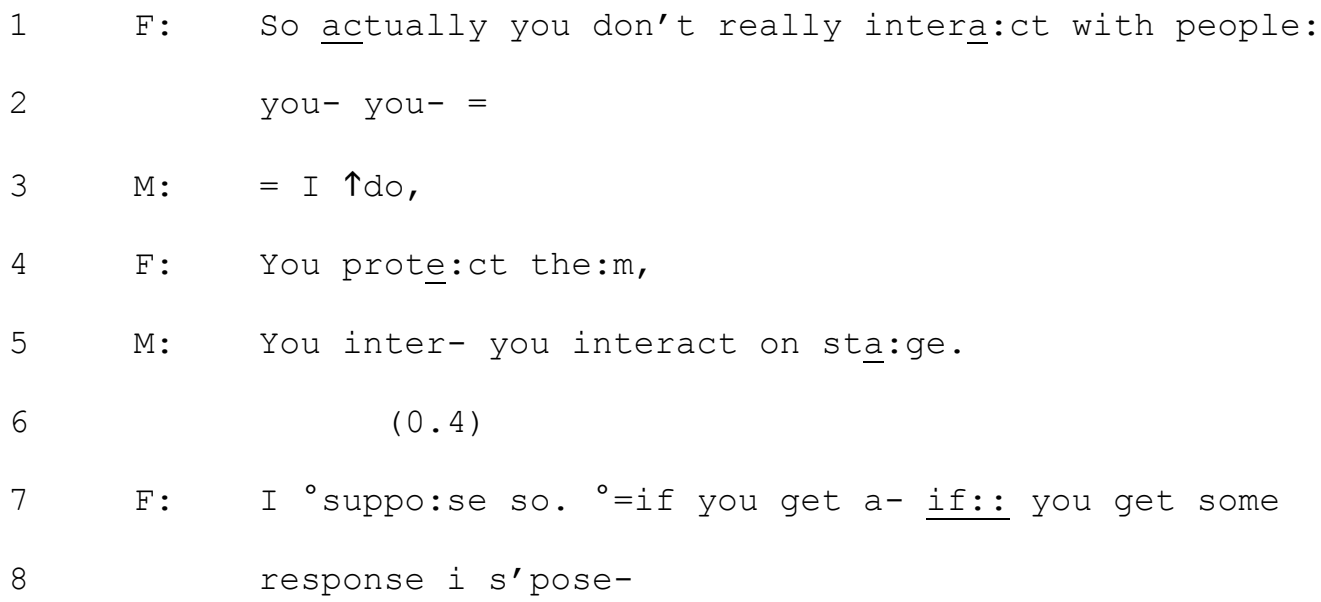

At line 7, F repairs her stance from 'if' to 'if::', with the first 'if' having no emphasis, whereas her second 'if::' is both emphasized and lengthened. The repair modifies F's stance in the turn being constructed, that that her assessment should be heard as conditional, and even sceptical, towards M's idea he gets a response from the audience whilst on stage. The key thing to note is that the repair is hearable by $\mathrm{M}$ and works to indicate what sort of turn $\mathrm{F}$ is formulating. That is, $\mathrm{F}$ cannot undo the first formulation of 'if' such that only the second is heard by M.

Let us compare Extract 16 to an online example. In Extract 17, Isla has been chatting to Callum. He has stated that he is logging off Facebook chat to watch a television programme called 'Secret Diary of a Call Girl', about a prostitute, starring the British actor Billie Piper. 
(17) [JM/IS6/F: 119-124]

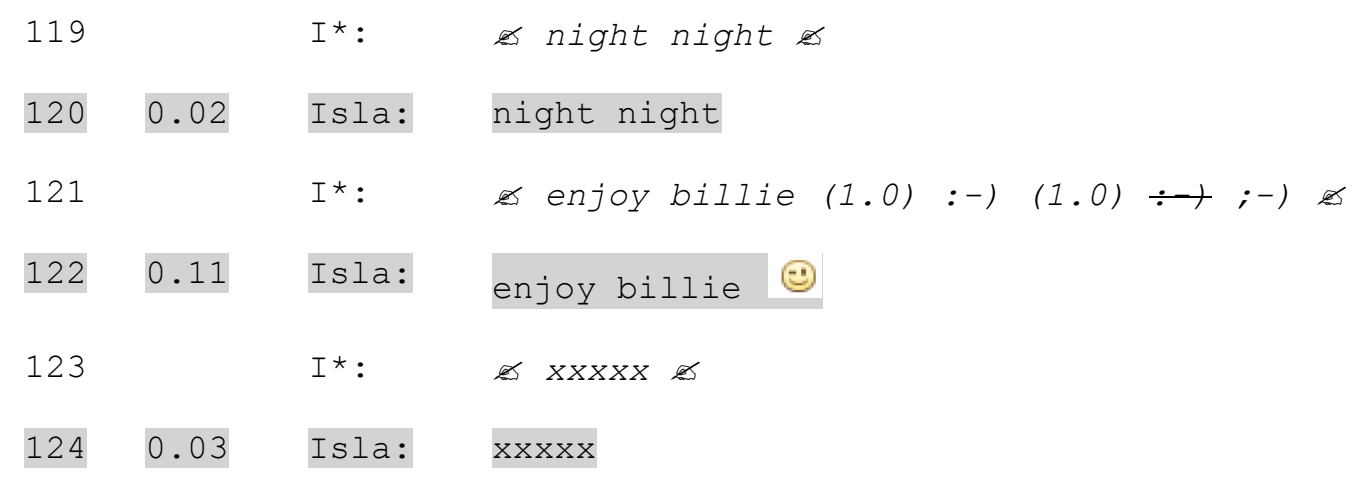

At line 119, Isla is progressing a closing sequence. At line 121 she initially types a 'smiley face' but then deletes it and repairs it to a 'wink'. Smilies can perform a number of functions in an interaction (Markman and Oshima, 2007) and may be (designedly) ambiguous in terms of their action (see Speer and Stokoe, forthcoming). The replacement (Schegloff, 2013)in Extract 17 repairs Isla's stance towards 'enjoy billie' from something like 'enjoyment of the programme' to a more 'salacious' orientation to the fact that the programme is about a call girl. What is clear, though, is that for Isla, there is sufficient difference in the potential inferences made available by a 'smiley face' and a 'wink' that the precise emoticon used becomes the object of repair.

In another extract, Callum requests that Isla teaches him how to throw the javelin. 
(18) [JM/IS5/F: 85-99]

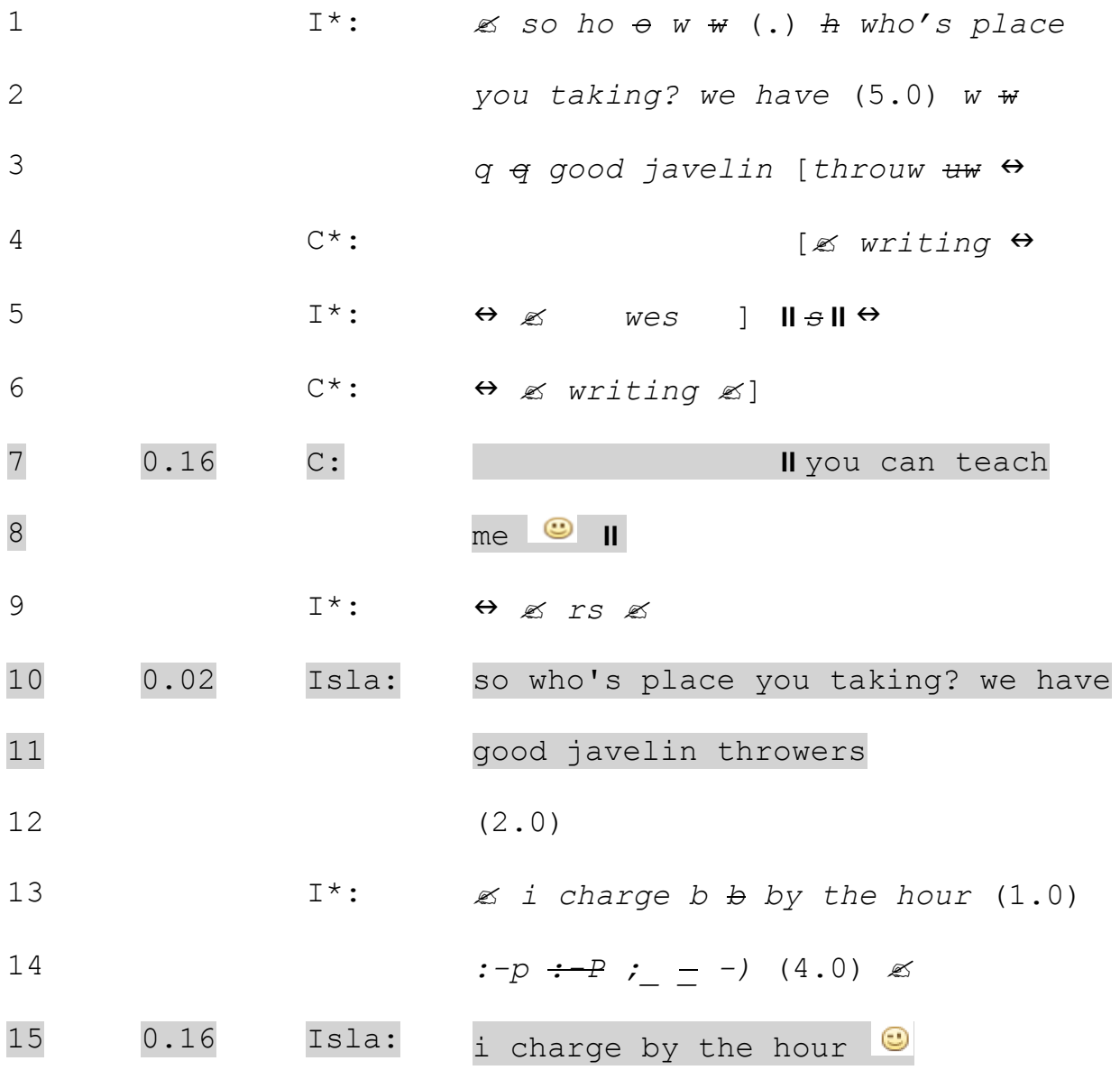

Isla constructs her response to Callum's request at lines 13 and 14. During message construction Isla begins to post a smiley with its tongue sticking out (:-P). However, she repairs this at line 14 to a 'wink'. As with Extract 17, we could gloss this as repairing the stance from a playful orientation to a flirtatious one. Such stances are, though, 'designedly ambiguous' (Speer and Stokoe, forthcoming; Stokoe, 2012) .We can simply 
say that, again, for Isla these two emoticons have different meanings for the action she is constructing. This repair is, of course, unavailable to Callum.

Related to repairs of stance are repairs of pronunciation. In Extract 19, Isla and Gavin have been discussing when Gavin - who is living in America - is going to visit Isla, who is based in the UK. The repair occurs at line 8 .

(19) [JM/IS14/B: 37-46]

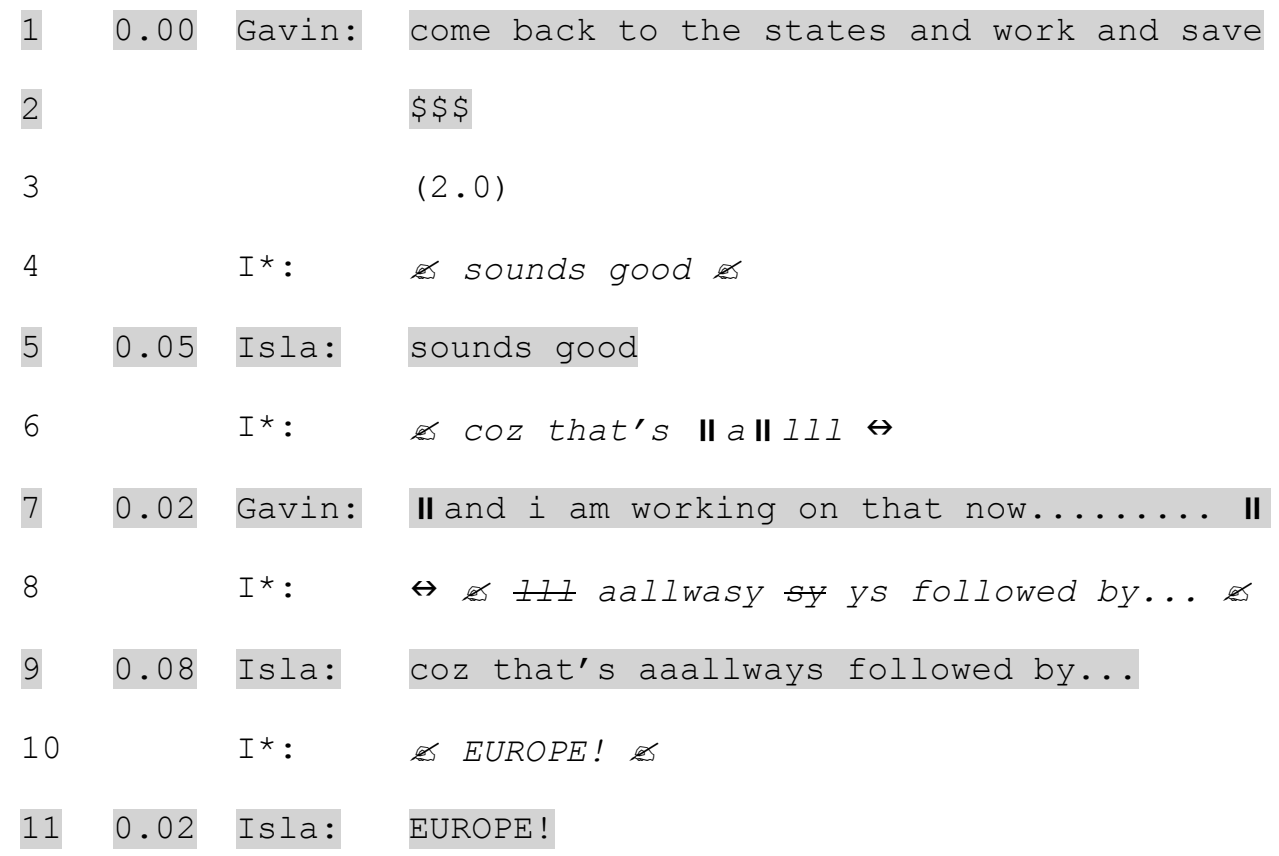

At line 6, Isla starts writing 'alll' but at line 8 she repairs it to 'aallways' orienting to the way Gavin should 'hear' this word. As with repairs of stance, we cannot tell precisely 
how Isla intends Gavin to 'hear' this. We merely know that for her there is a difference in meaning or emphasis of the words 'alllways' and 'aaallways', for which a repair operation is necessary. In spoken interaction, repairs of pronunciation are often otherinitiated (Jefferson, 1987). However, there are examples of self-initiated self-repair of pronunciation, as in the following example from a telephone call between Gordon and Dana.

(20) Holt [88U-1-08]

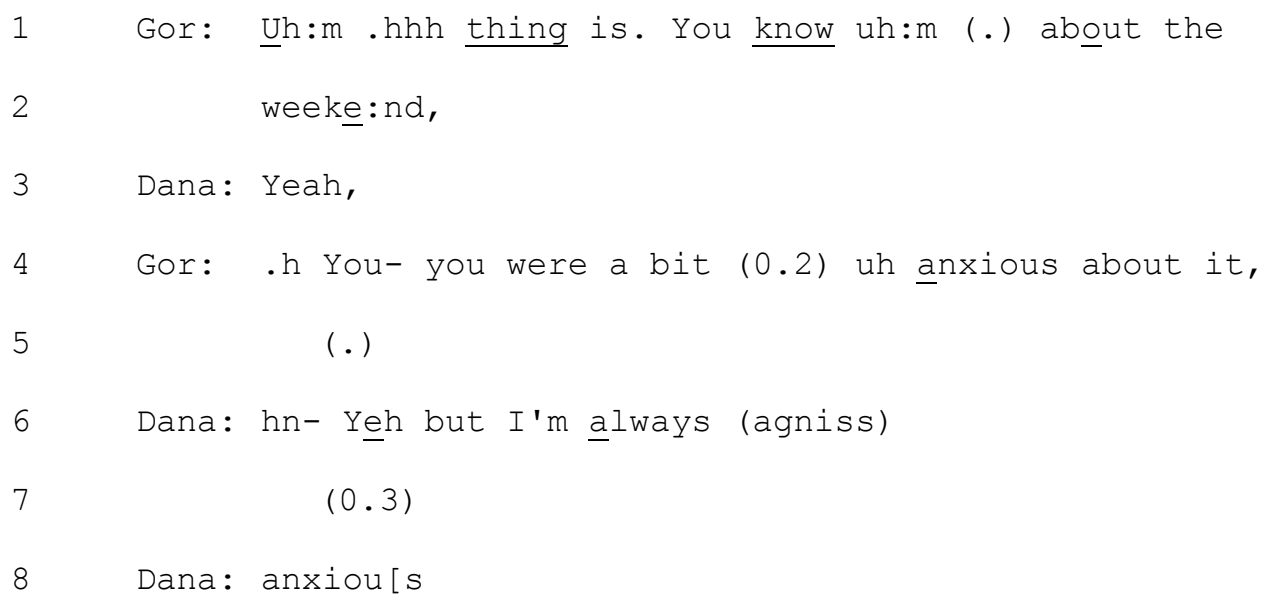

At line 6 Dana appears to say that she is always 'anxious' yet the pronunciation is not clear, and after a gap - which could indicate some trouble with Gavin's understanding of the word - she repeats the word, repairing the pronunciation. However, in this case there is a hearable error with Dana's pronunciation of the word, which could make it difficult for the recipient to know how to respond. In Extract 19 the trouble-source was 
the non-standard spelling of 'always', and yet it is not repaired to the correct spelling of the word, but rather to a different non-standard spelling. What Isla effectively does, then, is to repair the emphasis of the word, so that the 'aa' sound is more elongated. Therefore, this type of repair is not to repair a misspelling (as the visible repairs were), but rather is to repair the emphasis on the word.

The following example also demonstrates a change of emphasis, but repairs the 'prosody' rather than the 'pronunciation'. In this extract, Katie is discussing reporting a problem to a company and the difficulties she had in getting a response.

(21) [JM/KA14/B: 266-261]

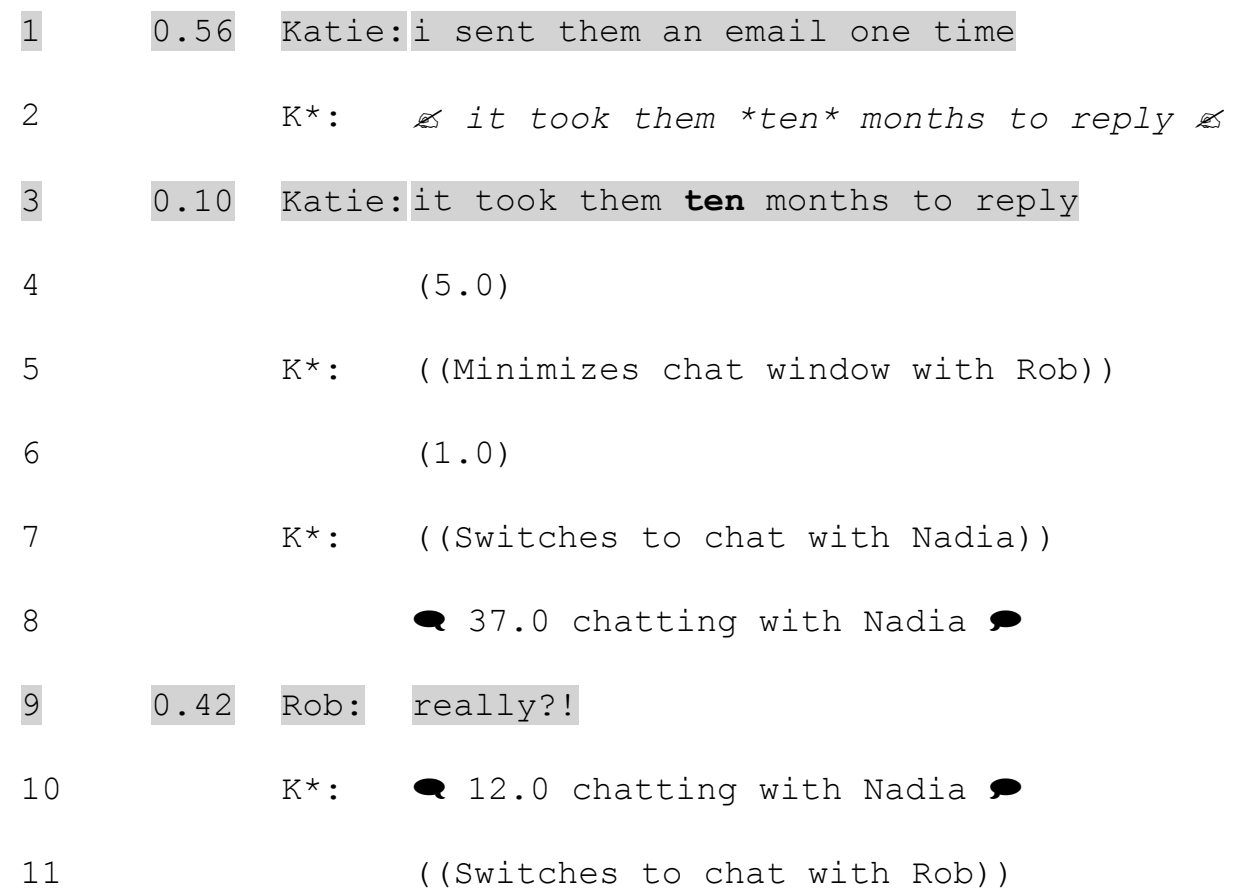


In online communication, it is possible to emphasise a word by emboldening it. In Facebook chat this is done by placing asterisks around the word during message construction (as Katie does in line 2); it will then appear in bold in the chat window. At line 12, Katie constructs her response to Rob's question 'really?!' at line 9. Note that the punctuation in Rob's turn indicates not only how this should be 'heard' but also some surprise or incredulity about Katie's turn at line 3. Thus, it also indicates Rob's stance towards the prior turn. Katie's turn at line 13 offers confirmation of this through the repetition of the word 'really'. Originally she writes 'really' with no emphasis, but then repairs it by adding asterisks so that it will appear in bold, suggesting a stress on the word. Again, then, this serves to indicate to the recipient how the word should be 'heard' and also the participants' stance.

We can see how 'pronunciation' and 'prosody' can be repaired during message construction, and that such repairs not only serve to indicate how words should be 'heard', but also the stance of the speaker. We have noted that the precise stance may not be easily analysed, partly because individuals may use the same emoticon in different ways. Again, this demonstrates that we must analyse online interactions in terms of what the participants do, rather than what the technology allows. More research 
with a larger corpus would start to unpack how participants themselves understand different emoticons or forms of pronunciation.

Whilst in most cases of message construction repair, recipients do not know that a repair has occurred; we end the analysis with a single deviant case, in which the person who has produced a repair during message construction orients to it in her chat.

(22) [JM/IS16/B: 269-272]

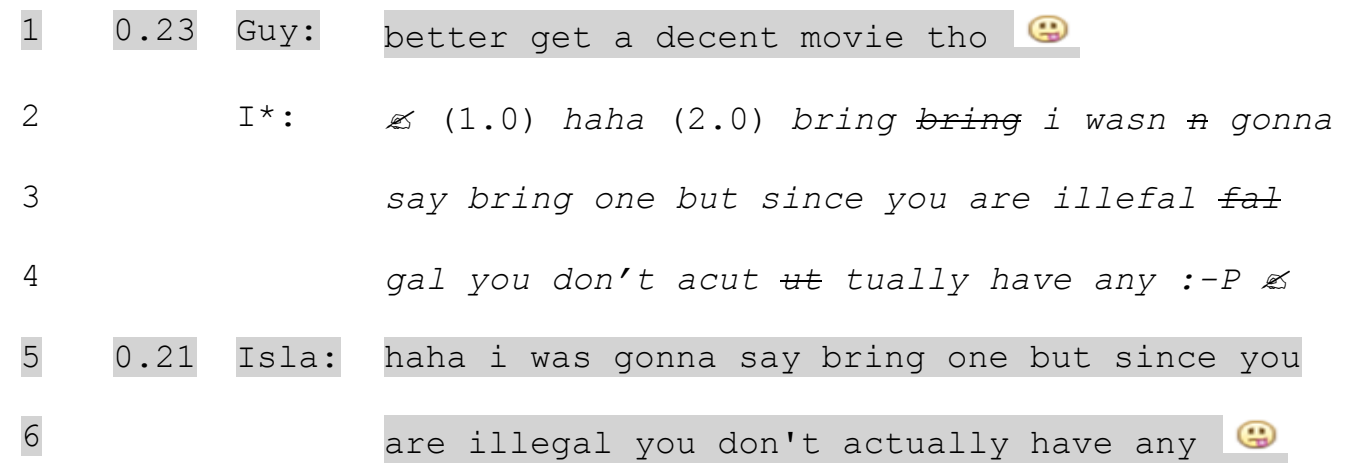

At line 2 Isla starts to write 'bring one' but then deletes this. She then repairs this to ' $\mathrm{i}$ was gonna say bring one', which she subsequently sends to the chat. In other words, she makes her repair interactionally relevant. As this does not occur in any other examples, we can ask why she does this particular action now. Isla and Guy are talking about getting together to watch a film. However, Guy can presumably not supply the film because he only (illegally) downloads them. Thus, Isla's repair during message construction attends to the potential trouble of requesting a movie from Guy, when she 
knows that he would not be able to grant that request (Drew et al., 2013). By mentioning the repair in her sent message, however, she makes Guy accountable for illegally downloading movies. However, note, that Guy does not know whether this repair actually occurred, and could presume that Isla is saying this simply to make him accountable for illegally downloading movies. Whereas in spoken interaction the repairs are hearable, can be oriented to and speakers may be held accountable for them, in online interaction it is the choice of the participant whether they reveal the repairs they make.

\section{Discussion}

This paper has shown that, and examined how, self-initiated self-repair occurs in online interaction, in both similar and contrasting ways to repair operations in spoken conversation. Two types of repair occurred: visible repair which could be seen and oriented to by both participants in the interaction, and message construction repairs, which were available only to a message's writer and occurred during message construction. We showed that visible repair occurred in some of the same sequential positions as in spoken conversation. However, the repairs were most commonly targeted at a trouble-source which is particular to written online interactions - spelling or typing errors. 
We also identified a form of repair that is unavailable to parties in spoken conversation; that is, message construction repair which occurs without recipients' knowledge. This is available due to the technological affordances of online interaction, and observable to us as analysts via the screen-capture methodology pioneered in this paper. The separation of message production and 'transmission' meant that, for example, the development of action in a turn, and the way action may be restarted, reformulated, and so on, was unavailable to recipients. This is a key affordance, because it means that, unlike in spoken interaction, online interlocutors are unaccountable for some interactional matters.

However, similar features of turn or message construction were repaired in online interaction as those found in talk, including repairs on stance, prosody, and action formation (see Schegloff, 2013). Thus participants in both online and spoken interaction are oriented to the same basic contingencies of maintaining intersubjectivity and building sequentially organized courses of action. This contradicts findings which suggest that repairs of writing only occur 'where the writer notices a discrepancy between text and intention' (Myhill and Jones, 2007: 34) or where there are 'errors and other perceived inadequacies' (Crystal, 2001: 27). This article has instead shown that writers in Facebook chat repair their messages when there is no error, instead repairing the action or sequential implications of their turn. We also suggest that our observations support the conversation analytic argument that features of talk like repair, laughter and 
emotional conduct do not 'leak' into interaction accidentally, but are designed and positioned for action (e.g. Hepburn, 2004; Potter and Hepburn, 2010). We also find that in examining message construction repair, we can identify what self-repairs achieve interactionally. In other words, as Drew et al. (2013: 74) note,

the original version - that is the version that the speaker begins, though sometimes does not complete - can be compared with the eventual version, the repair, to identify in what ways the speaker has modified, altered or adjusted their turn to deal with something other than a factual error

As our paper has shown, in Facebook chat, participants do extensive repairs during message construction, which can be compared with the sent version, in order to identify the various actions oriented to by doing that repair.

It is also notable that the preference for self-correction holds in online interaction (Schegloff et al., 1977). As in spoken interaction, 'a great many troubles occur, and are managed within, a current turn - before a speaker reaches its first possible completion' (Hayashi et al, 2013: 12). In other words, participants orient to the preference for completing a repair as early as possible after the trouble source. In online interaction the earliest point is during message construction. This, again, suggests that participants orient to similar preferences as those in spoken interaction, but that the 
affordances of the medium mean that repair completed at the earliest point after the trouble-source also happen to be unavailable to the recipient.

This paper also demonstrates empirically the affordances of the online medium and how these are oriented to by participants. While the technology allows for the editing of messages during construction, the ways in which participants actually do this has never been examined in a naturally-occurring setting. Therefore, we have shown that while participants $d o$ edit and delete messages prior to sending, they do not take time to, for example, check spelling before sending messages. This has clear implications for understanding how a technological affordance can, and does, affect an interaction.

The analysis presented in the paper has implications for the study of, and for comparisons between, online and spoken interaction and, perhaps, for the study of writing-as-interaction. On the one hand, conversation analytic findings and conventions do not, perhaps unsurprisingly, map directly onto online interaction. Although we found that, for example, the positioning of some repairs was similar to those found in spoken conversation; there was no one-to-one correspondence. It is, therefore, necessary to examine online interactions as an adaptation of existing speech-exchange systems. Participants in virtual worlds do not create entirely new ways of communicating, but rather recreate and adapt features of communication from the offline world (Benwell and Stokoe, 2006). And so, we propose that the study of online interaction treats it as a 
speech-exchange system with its own normative rules, which are adapted from, and show similar orientations to, the speech exchange system of ordinary, mundane interaction. This paper has provided preliminary evidence for some of the 'systematic ways' in which online interactions differ from ordinary talk. We hope that this paper contributes to dismantling stereotypes about distinctions between spoken and online interaction, as well as raising the visibility of interaction-based studies of what is surely a permanent, omnipresent form for social interaction and social life.

\section{Appendix: Full list of transcription symbols}

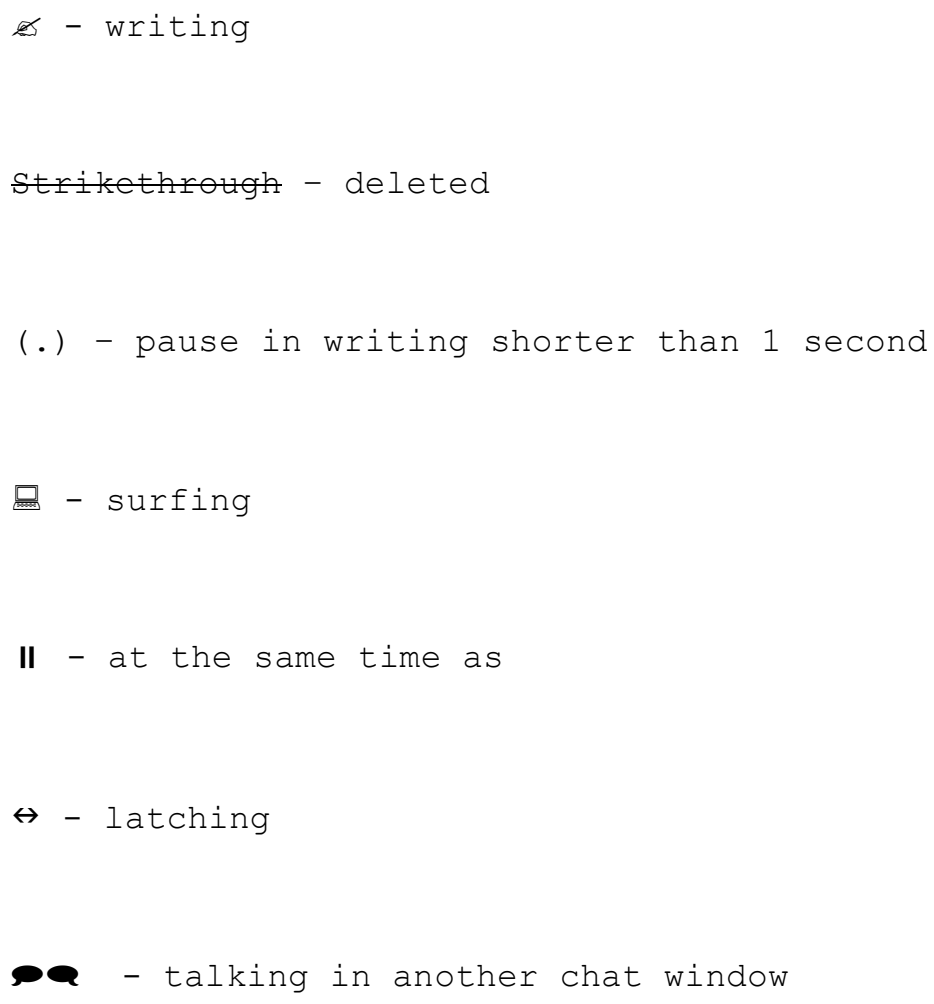




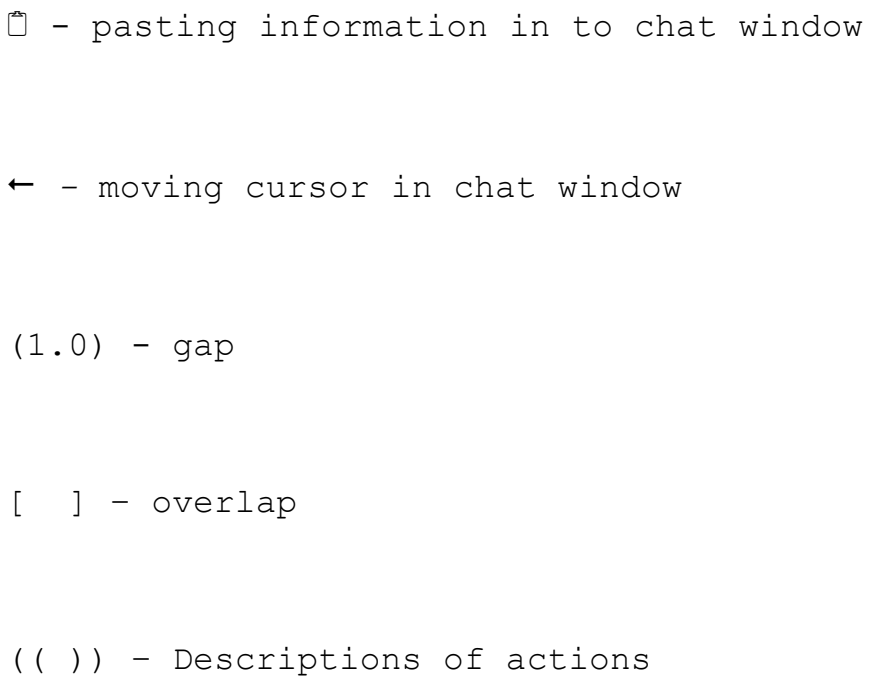

\section{Acknowledgements}

We acknowledge Anita Pomerantz and an anonymous reviewer for their very helpful comments on an earlier draft of this paper. We also thank Charles Antaki for some insightful observations on some of our data extracts. This work was supported by the Economic and Social Research Council [Grant number: ES/I903321/1] 


\section{References}

Antaki C, Ardévol E, Núñez F and Vayreda A (2005) 'For she who knows who she is': Managing Accountability in Online Forum Messages. Journal of Computer-Mediated Communication 11(1): Retrieved http://jcmc.indiana.edu/vol11/issue1/antaki.html.

Benwell B and Stokoe E (2006) Discourse and Identity. Edinburgh: Edinburgh University Press.

Berglund TÖ (2009) Disrupted turn adjacency and coherence maintenance in instant messaging conversations. Language@Internet 6(2): Retrieved www.languageatinternet.de/articles/2009/2106/Berglund.pdf/.

Bolden GB, Mandelbaum J and Wilkinson S (2012) Pursuing a response by repairing an indexical reference. Research on Language and Social Interaction 45(2): 137-155.

Brown C (2009) WWW.HATE.COM: White Supremacist Discourse on the Internet and the Construction of Whiteness Ideology. The Howard Journal of Communications 20(2): 189-208.

Cherny L (1999) Conversation and Community: Chat in a Virtual World. Stanford, CA;: Center for the Study of Language and Information.

Collister LB (2011) *-Repair in online discourse. Journal of Pragmatics 23(3): 918-921. 
Crystal D (2001) Language and the Internet. Cambridge: Cambridge University Press.

Drew P, Walker T and Ogden R (2013) Self-repair and action construction. In: Hayashi M, Raymond G and Sidnell J (eds) Conversational repair and human understanding. Cambridge: Cambridge University Press, 71-94.

Edwards D (2005) Moaning, whinging and laughing: The subjective side of complaints. Discourse Studies 7(1): 5-29.

Epperson TW and Zemel A (2008) Reports, Requests, and Recipient Design: The Management of Patron Queries in Online Reference Chats. Journal of the American Society for Information Science and Technology 59(14): 2268-2283.

Flinkfeldt M (2011) 'Filling one's days': Managing sick leave legitimacy in an online forum. Sociology of Health \& Illness 33(5): 761-776.

Garcia AC and Jacobs JB (1999) The eyes of the beholder: Understanding the turntaking system in quasi-synchronous computer-mediated communication. Research on Language and Social Interaction 32(4): 337-367.

Gibson W (2009) Intercultural Communication Online: Conversation Analysis and the Investigation of Asynchronous Written Discourse. Forum Qualitative Sozialforschung/Forum: Qualitative Social Research 10(1). 
Goodwin C (2007) Participation, stance and affect in the organization of activities.

Discourse \& Society 18(1): 53-73.

Greenfield PM and Subrahmanyam K (2003) Online discourse in a teen chatroom: New codes and new modes of coherence in a visual medium. Journal of Applied Developmental Psychology 24(6): 713-738.

Hepburn A (2004) Crying: Notes on description, transcription and interaction. Research on Language and Social Interaction 37(3): 251-290.

Hepburn A, Wilkinson S and Shaw R (2012) Repairing self and recipient reference. Research on Language and Social Interaction 45(2): 175-190.

Heritage J (2005) Conversation Analysis and Institutional Talk. In: Fitch KL and Sanders RE (eds) Handbook of Language and Social Interaction. Mahwah, NJ: Erlbaum, 103-147.

Herring SC (1999) Interactional coherence in CMC. Journal of Computer-Mediated Communication 4(4): Retrieved http://www.ascusc.org/jcmc/vol4/issue4/herring.html.

Hester S and Francis D (2000) Ethnomethodology, conversation analysis and 'institutional talk'. Text 20(3): 391-413. 
Holtz P and Wagner W (2009) Essentialism and attribution of monstrosity in racist discourse: Right-wing Internet postings about Africans and Jews. Journal of Community \& Applied Social Psychology 19(6): 411-425.

Hayashi M, Raymond G and Sidnell, J (2013) Conversational repair and human understanding: An introduction. In: Hayashi M, Raymond G and Sidnell J (eds) Conversational repair and human understanding. Cambridge: Cambridge University Press, 1-40.

Horne J and Wiggins S (2009) Doing being 'on the edge': Managing the dilemma of being authentically suicidal in an online forum. Sociology of Health and Illness 31(2): 170-184.

Hutchby I (2001) Conversation and Technology. Cambridge: Polity Press.

Hutcby I (2003) Affordances and the analysis of technologically-mediated interaction. Sociology 37: 581-589.

Hutchby I and Wooffitt R (1998) Conversation Analysis: Principles, Practices and Applications. Cambridge: Polity Press. 
Jefferson G (2004) Glossary of transcript symbols with an introduction. In: Lerner GH (ed) Conversation Analysis: Studies from the First Generation. Philadelphia: John Benjamins, 13-23.

Jefferson G (1987) On exposed and embedded correction in conversation. In: Button G and Lee JRE (eds) Talk and Social Organisation. Clevedon: Multilingual Matters, 86100.

Jefferson G (1978) Sequential aspects of storytelling in conversation. In: Schenkein J (ed) Studies in the Organization of Conversational Interaction. New York: Academic Press, 219-248.

Jefferson G (1974) Error correction as an interactional resource. Language in Society 2: 181-199.

Jefferson G, Sacks H and Schegloff EA (1987) Notes on laughter in the pursuit of intimacy. In: Button G and Lee JRE (eds) Talk and Social Organisation. Clevedon: Multilingual Matters, 152-205.

Kiztinger C, Shaw R and Toerien M (2012) Referring to persons without using a fullform reference: Locally initial indexicals in action. Research on Language and Social Interaction 45(2): 116-136. 
Lamerichs J and te Molder HFM (2003) Computer-mediated communication: From a cognitive to a discursive model. New Media \& Society 5(4): 451-473.

Lerner GH and Kitzinger C (2007) Extraction and aggregation in the repair of individual and collective self-reference. Discourse Studies 9(4): 526-557.

Lerner GH, Bolden GB, Hepburn A and Mandelbaum J (2012) Reference recalibration repairs: Adjusting the precision of formulations for the task at hand. Research on Language and Social Interaction 45(2): 191-212.

Liddicoat AJ (2007) An Introduction to Conversation Analysis. London: Continuum. Local J and Walker G (2008) Stance and affect in conversation: On the interplay of sequential and phonetic resources. Text \& Talk 28(6): 723-747.

Macbeth D (2004) The relevance of repair for classroom correction. Language in Society 33(5): 703-736.

Markman KM (2005) To send or not to send: Turn construction in computer-mediated chat. In: Twelfth Annual Symposium about Language and Society, Austin, Texas, 16-18 April 2005, pp.115-124.

Markman KM (2009) 'So what shall we talk about': Openings and closings in chatbased virtual meetings. Journal of Business Communication 46(1): 150-170. 
Markman KM and Oshima S (2007) Pragmatic play? Some possible functions of English emoticons and Japanese Kaomoji in computer mediated discourse. In: Proceedings of the Association of Internet Researchers Annual Conference 8.0: Let's play!, Vancouver, B.C, 18 October 2007.

Martin C and Sahlström F (2010) Learning as longitudinal interactional change: From other-repair to self-repair in physiotherapy treatment. Discourse Processes 47(8): 668697.

Myhill D and Jones S (2007) More than just error correction. Written Communication 24(4): 323-343.

Potter J and Hepburn A (2010) Putting aspiration into words: 'Laugh particles', managing descriptive trouble and modulating action. Journal of Pragmatics 42: 15431555.

Rawclaw J (2008) Two patterns for conversational closings in instant message discourse. Colorado Research in Linguistics 21: 1-21.

Rintel ES, Mulholland J and Pittam J (2001) First things first: Internet relay chat openings. Journal of Computer Mediated Communication 6(3): Retrieved: http://onlinelibrary.wiley.com/doi/10.1111/j.1083-6101.2001.tb00125.x/full. 
Schegloff, EA (2013) Ten operations in self-initiated, same turn repair. In: Hayashi M, Raymond G and Sidnell J (eds) Conversational repair and human understanding.

Cambridge: Cambridge University Press, 71-70.

Schegloff EA (2007) Sequence Organization in Interaction: A Primer in Conversation Analysis. Cambridge: Cambridge University press.

Schegloff EA (2006) Repair after Next Turn: The last structurally provided defense of intersubjectivity in conversation. In: Drew P and Heritage J (eds) Conversation Analysis, Volume 1: Turn-Taking and Repair. London: Sage Publications, 215-266.

Schegloff EA (1997) Third Turn Repair. In: Guy GG, Feagin C, Schiffrin D and Baugh J (eds) Towards a Social Science of Language 2. Amsterdam: John Benjamins, 31-40.

Schegloff EA (1987) Recycled turn beginnings: A precise repair mechanism in conversation's turn-taking organisation. In: Button G and Lee JRE (eds) Talk and Social Organisation. Clevedon: Multilingual Matters, 70-85.

Schegloff EA, Jefferson G and Sacks H (1977) The preference for self-correction in the organization of repair in conversation. Language 53(2): 361-382.

Schönfeldt J and Golato A (2003) Repair in chats: A conversation analytic approach. Research on Language and Social Interaction 36(3): 241-284. 
Sidnell J (2010) Conversation Analysis: An Introduction. Chichester, West Sussex; Malden, MA: Wiley-Blackwell.

Sneijder P and te Molder HFM (2005) Moral Logic and Logical Morality: Attributions of Responsibility and Blame in Online Discourse on Veganism. Discourse \& Society 16(5): 675-696.

Speer SA and Stokoe E (Forthcoming) Flirting: A designedly ambiguous action. Manuscript in Preparation.

Stivers T (2008) Stance, alignment and affiliation during storytelling: When nodding is a token of affiliation. Research on Language and Social Interaction 41(1): 31-57.

Stokoe E (2012) Moving forward with membership categorization analysis: Methods for systematic analysis. Discourse Studies 14(3): 277-303.

Stokoe E (2011) 'Girl - woman - sorry!': On the repair and non-repair of consecutive gender categories. In: Speer SA and Stokoe E (eds) Conversation and Gender. Cambridge: Cambridge University Press, 84-111.

Stokoe E (2010) ‘Have you been married or...?' Eliciting and accounting for relationship histories in speed-dating encounters. Research on Language and Social Interaction 43(3): 260-282. 
Stommel W (2008) Conversation Analysis and Community of Practice as Approaches to Studying Online Community. Language@ Internet 5: Article 5. Retrieved http://www.languageatinternet.org/articles/2008/1537

Stommel W and Koole T (2010) The online support group as a community: A microanalysis of the interaction with a new member. Discourse Studies 12(3): 357-378.

Vayreda A and Antaki C (2009) Social Support and Unsolicited Advice in a Bipolar Disorder Online Forum. Qualitative Health Research 19(7): 931-942.

Werry CC (1996) Linguistic and interactional features of Internet Relay Chat. In: Herring SC (ed) Computer-Mediated Communication: Linguistic, Social and CrossCultural Perspectives. Amsterdam: John Benjamins, 47-64.

Wilkinson S and Weatherall A (2011) Insertion Repair. Research on Language and Social Interaction 44(1): 65-91. 